A thesis submitted to the Faculty of Graduate and Postdoctoral Affairs in partial fulfillment of the requirements for the degree of Master of Applied Science in Electrical and Computer Engineering

Ottawa-Carleton Institute for Electrical and Computer Engineering (OCIECE)

Department of Systems and Computer Engineering

Carleton University

Ottawa, Ontario, Canada, K1S 5B6

September 2011

(C) Copyright 2011, Nazia Ahmad 


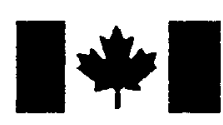

\author{
Library and Archives \\ Canada \\ Published Heritage \\ Branch \\ 395 Wellington Street \\ Ottawa ON K1A ON4 \\ Canada
}

Bibliothèque et

Archives Canada

Direction du

Patrimoine de l'édition

395 , rue Wellington

Ottawa ON K1A ON4

Canada
Your file Votre référence
ISBN: $978-0-494-83060-4$
Our file Notre référence
ISBN: $978-0-494-83060-4$
NOTICE:

The author has granted a nonexclusive license allowing Library and Archives Canada to reproduce, publish, archive, preserve, conserve, communicate to the public by telecommunication or on the Internet, loan, distribute and sell theses worldwide, for commercial or noncommercial purposes, in microform, paper, electronic and/or any other formats.

The author retains copyright ownership and moral rights in this thesis. Neither the thesis nor substantial extracts from it may be printed or otherwise reproduced without the author's permission.
AVIS:

L'auteur a accordé une licence non exclusive permettant à la Bibliothèque et Archives Canada de reproduire, publier, archiver, sauvegarder, conserver, transmettre au public par télécommunication ou par l'Internet, prêter, distribuer et vendre des thèses partout dans le monde, à des fins commerciales ou autres, sur support microforme, papier, électronique et/ou autres formats.

L'auteur conserve la propriété du droit d'auteur et des droits moraux qui protège cette thèse. Ni la thèse ni des extraits substantiels de celle-ci ne doivent être imprimés ou autrement reproduits sans son autorisation.
In compliance with the Canadian Privacy Act some supporting forms may have been removed from this thesis.

While these forms may be included in the document page count, their removal does not represent any loss of content from the thesis.
Conformément à la loi canadienne sur la protection de la vie privée, quelques formulaires secondaires ont été enlevés de cette thèse.

Bien que ces formulaires aient inclus dans la pagination, il n'y aura aucun contenu manquant. 
The undersigned recommend to the Faculty of Graduate and Postdoctoral Affairs acceptance of the thesis

\title{
Enhanced Topology Control Interference Aware Channel Assignment Algorithm
}

\author{
submitted by \\ Nazia Ahmad, B. Tech. \\ in partial fulfillment of the requirements for \\ the degree of Master of Applied Science in Electrical and Computer \\ Engineering
Chair, Howard Schwartz, Department of Systems and Computer Engineering

Thesis Supervisor, Prof. Roshdy H. M. Hafez 


\begin{abstract}
Minimizing co-channel interference is a key issue for channel assignment in Multi-Radio Multi-Channel (MRMC) Wireless Mesh Networks (WMN). Topologycontrolled Interference-aware Channel-assignment Algorithm (TICA) is a new system for channel assignment which uses one-way interference range edge coloring during channel assignment. However, two links that are within the interference range of each other could be assigned the same channel, which would lead to interference and reduction in network throughput. In this thesis, we study the above problem in the context of TICA. We propose and evaluate an enhanced version of TICA, named e-TICA. The proposed scheme solves the interference problem by using two-way interference range edge coloring. Also, in e-TICA, the presence of relatively long links in some topologies leads to conflicting channel assignments, which results in contention among nodes for channel access. To remedy this problem, we propose a modified version of e-TICA named eTICA2. This new scheme utilizes a minimum spanning tree rooted at the gateway to reduce conflict in channel assignments by eliminating long links, hence improving the network throughput. Additionally, in order to utilize the four radios of the gateway, eTICA2 enables the gateway to build a minimum spanning tree from its closest neighbors, thus distributing the traffic load among the links originating at the gateway. We use computer simulations to evaluate and compare the performance of all three channel assignment algorithms, namely TICA, e-TICA and e-TICA2. Simulation results indicate that e-TICA outperforms the original TICA scheme in most topologies investigated. Furthermore, e-TICA2 outperforms both of them in most cases.
\end{abstract}




\section{Acknowledgements}

"In the name of Allah, most Gracious, most Compassionate"

I owe my deepest gratitude to Prof. Hafez for his valuable guidance and patience with me throughout my research work. This thesis would not have been possible without his encouragement and supervision.

I am also grateful to the defense committee members Prof. Michel Barbeau, Prof. Richard Yu and Prof. Ioannis Lambadaris for their valuable feedback and comments which served as a means of enhancing my thesis.

I am indebted to my colleague Aizaz who has supported me in numerous ways. He assisted me at every step providing valuable feedback and giving up much of his time to discuss and proofread my thesis. I must make a special mention of my colleagues Talha and Yasser whose willingness to help and give suggestions was extremely useful for the progress of this thesis.

I am grateful to my husband Meraj who was always been there for me as a pillar of support during my research work. I would also like to thank my adorable daughters Nabiha and Maahira whose unconditional love and affection gave me the strength to keep going despite all adversities.

I would also like to express my deepest gratitude to my friends and family all over the world especially to my mother and father-in-law for their love, prayers and encouragement during the course of this research work. I would like to seize this opportunity to appreciate my aunts, uncles, cousins, brothers, brothers-in-law and sistersin-law for their affection and words of encouragement which boosted my morale. 


\section{Table of Contents}

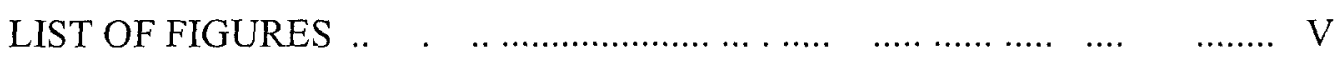

LIST OF TABLES ......................................................................... VII

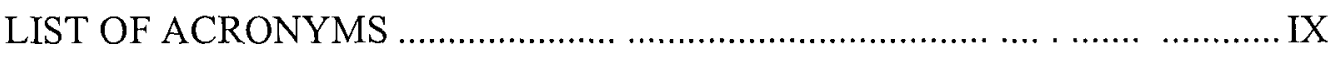

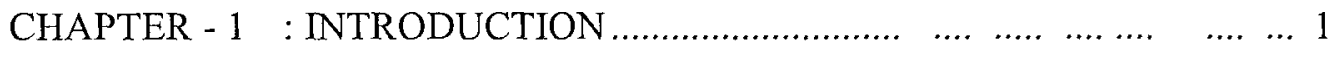

11 INTRODUCTION 1

12 WIRELESS MESH NETWORKS 1

13 MOtiVATiON 3

14 CONTRIBUTIONS 3

15 Thesis Organization $\quad 4$

CHAPTER - $2 \cdot$ BACKGROUND AND RELATED LITERATURE $\quad \ldots \quad 5$

21 INTRODUCTION 5

22 IEEE $80211 \times$ PROTOCOLS 5

221 IEEE 80211

222 IEEE $80211 a+6$

223 IEEE $80211 b \quad 6$

224 IEEE $802 \mathrm{llg}$. 6

23 MEDIUM ACCESS CONTROL (MAC) LAYER 6

231 PCF 6

$232 D C F \quad 7$

24 Related Literature 10

241 Channel Assignment Schemes 10

242 Topology Control Schemes 11

243 Fatrness in wireless networks $\quad 12$

$\begin{array}{lll}25 & \text { TICA } & 13\end{array}$

26 INTERFERENCE MODELING

CHAPTER - $3 \quad$ : PROBLEM STATEMENT ...................................... 16

31 INTRODUCTION $\quad$. $\quad 16$

32 ISSUES WITH TICA 16

33 Problem Statement e-TICA and e-TICA2 . 17

$\begin{array}{lll}331 & e-T I C A & 17\end{array}$

332 e-TICA2 18 
41 INTRODUCTION 19

42 TICA 20

421 Topology control phase 20

422 Connectivity phase 21

423 Minimum Power Shortest Path Tree 24

424 Channel Asstgnment phase 25

43 E-TICA 28

431 An accurate channel assignment algorthm $\quad 29$

44 E-TICA2 32

441 Reduction in Interference Mintmum Spanning Tree 32

442 Reduction in congestion Uttlizing the four radios of the $G W$

CHAPTER - 5 SIMULATION RESULTS AND EVALUATION 37

51 INTRODUCTION 37

52 Simulation 37

521 Simulation Description 37

522 Simulatton Parameters 38

523 Simulation Assumptions 38

53 Performance Metrics for e-TICA and e-TICA2 39

54 Accurate Channel Assignment e-TiCA 39

541 Results for Random topologies 40

542 Results for Controlled Random topologies 45

55 REDUCING INTERFERENCE AND CONGESTION ETICA2 49

551 Random Topologies 49

552 Controlled Random Topologtes $\quad 54$

56 GRID TOPOLOGY 58

561 Performance evaluation of TICA

562 Performance evaluation of e TICA 58

563 Performance evaluation of e-TICA2 58

57 CONSOLIDATION OF THE RESULTS $\quad 60$

CHAPTER - 6 CONCLUSION AND FUTURE WORK 61

61 CONCLUSIONS 61

62 DisCUSSION 61

63 TRADE-OFFS 62

64 FUTURE WORK 62 
REFERENCES

63

APPENDIX-A: SPT FOR RANDOM TOPOLOGIES

65

APPENDIX-B: SPT FOR CONTROLLED RANDOM TOPOLOGIES 66

APPENDIX-C: MST FOR RANDOM TOPOLOGIES 67

APPENDIX-D: MST FOR CONTROLLED RANDOM TOPOLOGIES 68 


\section{List of Figures}

Figure 1: Wireless Mesh Network ................................................................. 2

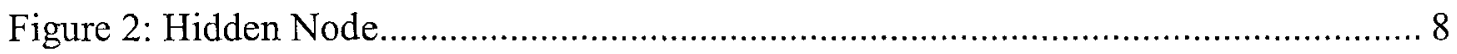

Figure 3: Exposed Node................................................................................. 8

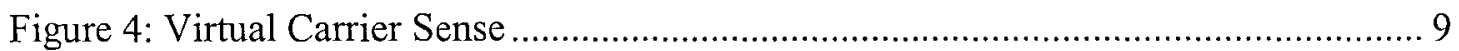

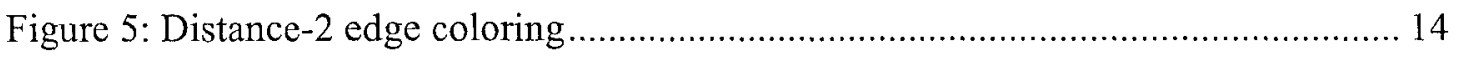

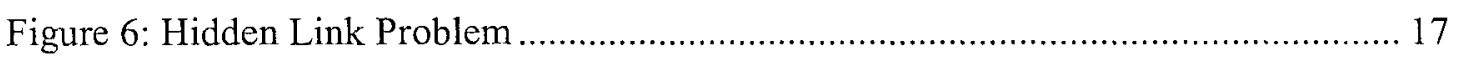

Figure 7: Maximum Power Transmission Range of Node 1 ..................................... 20

Figure 8: Connectivity Graph 'select 2 for less than 2' TCA ..................................... 23

Figure 9: Connectivity Graph ‘select 3 for less than 3' TCA ….................................. 23

Figure 10: Shortest Path Tree from GW (Node 15) to all other nodes ........................... 24

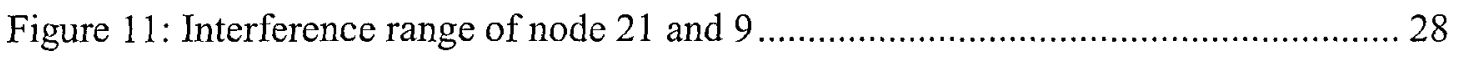

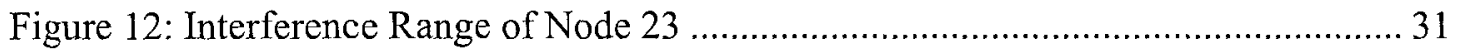

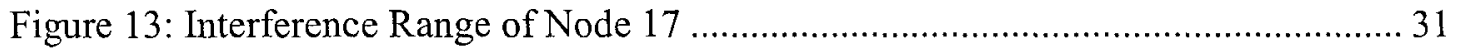

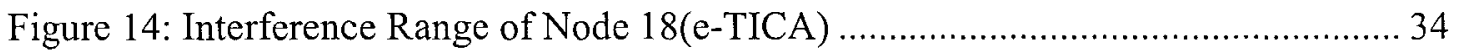

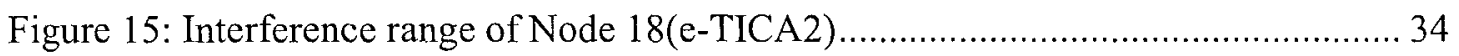

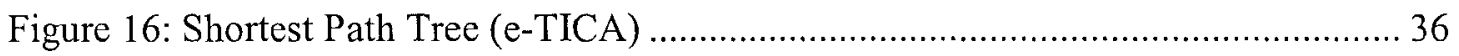

Figure 17: Minimum Spanning Tree (e-TICA2) …............................................... 36

Figure 18: Comparison of Throughput Ratio for 25 Random Topologies ..................... 43

Figure 19: Comparison of Fairness Ratio for 25 Random Topologies ........................... 43

Figure 20: Jain's Fairness Index for 25 Random Topologies ...................................... 44

Figure 21: Comparison of Throughput Ratio for 25 Controlled Random Topologies ..... 47

Figure 22: Comparison of Fairness Ratio for 25 Controlled Random Topologies........... 47

Figure 23: Jain's Fairness Index for 25 Controlled Random Topologies ........................ 48

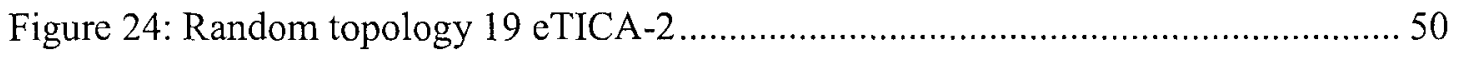

Figure 25: Comparison of Throughput Ratio of 25 Random Topologies........................ 52

Figure 26: Comparison of Fairness ratio of 25 Random Topologies ............................. 52

Figure 27: Fairness Index for all 3 CAAs for Random Topologies................................ 53

Figure 28: Comparison of Throughput Ratio of 25 Controlled Random Topologies...... 56 
Figure 29: Comparison of Fairness ratio of 25 Controlled Random Topologies 56

Figure 30: Fairness Index for all 3 CAAs for Controlled Random topologies................ 57 


\section{List of Tables}

Table 1: Maximum Power Transmission Range neighbors of Node 1 ............................ 21

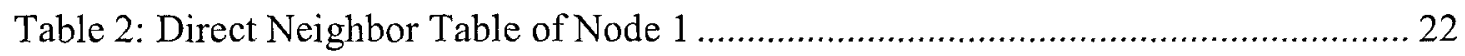

Table 3: Link Ranking and Channel Allocation ........................................................ 26

Table 4: Comparison of the channel assignment done by TICA and e-TICA ................ 30

Table 5: Physical Layer Parameters................................................................................ 38

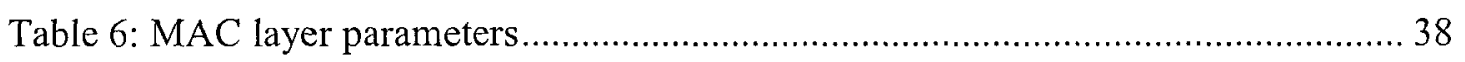

Table 7: LICs found by TICA and e-TICA for 25 Random Topologies ....................... 42

Table 8: Results for number of LICs for 25 Random Topologies ................................. 42

Table 9: Results for Throughput Ratio for 25 Random Topologies ............................ 43

Table 10: Results for Fairness Ratio of 25 Random Topologies .................................... 43

Table 11: Results for Jain's Fairness Index for Random Topologies ............................. 44

Table 12: LICs found by TICA and e-TICA for 25 Controlled Random Topologies ...... 46

Table 13: Results for number of LICs for Controlled Random Topologies ................... 46

Table 14: Results for Throughput Ratio of Controlled Random Topologies .................. 47

Table 15: Results for Fairness ratio of Controlled Random Topologies ....................... 47

Table 16: Results for Jain's Fairness Index ............................................................ 48

Table 17: LICs found in 25 Random Topologies by TICA, e-TICA and e-TICA2 ........ 51

Table 18: Results for number of LICs for Random Topologies ................................... 51

Table 19: Results for Throughput Ratio for Random Topologies ............................... 52

Table 20: Results for Fairness Ratio for Random Topologies.................................... 52

Table 21: Results for Jain`s Fairness Index for Random Topologies ............................. 53

Table 22: LICs found in 25 Controlled Random Topologies by TICA, e-TICA and e-

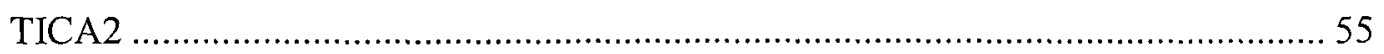

Table 23: Results for number of LICs for Controlled Random Topologies ................... 55

Table 24: Results for Throughput Ratio for Controlled Random Topologies .................. 56

Table 25: Results for Fairness Ratio for Controlled Random Topologies......................56

Table 26: Results for Jain`s Fairness Index for Controlled Random Topologies............ 57

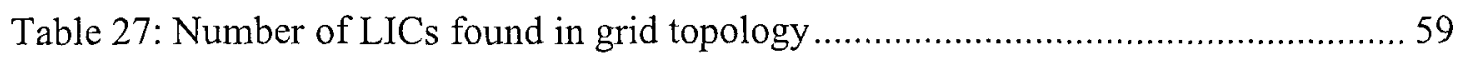




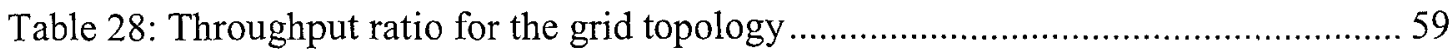

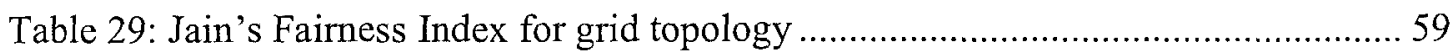

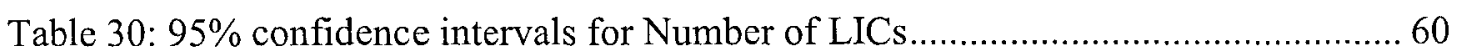

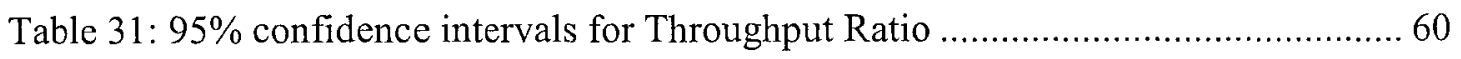

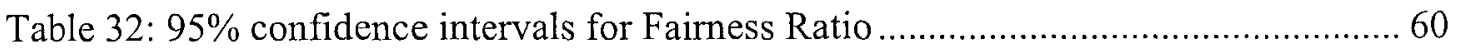




\section{List of Acronyms}

AP

BFS-CA

CAA

CLICA

CRT

CS

$\mathrm{CSMA} / \mathrm{CA}$

CTS

DCF

DIFS

DNT

DSSS

ECLICA

e-TICA

FHSS

$F_{J}$

$\mathrm{F}_{\mathrm{R}}$

GW

IR

JRCA

LIC

LMST

MAC

$\mathrm{MC}$

MCAIR

MCAR

MMAC
Access Point

Breadth First Search Channel Assignment

Channel Assignment Algorithm

Connected Low Interference Channel Assignment Algorithm

Controlled Random Topology

Carrier Sensing Range

Carrier Sense Multiple Access with Collision Avoidance

Clear to Send

Distributed Coordination Function

Distributed Inter Frame Space

Direct Neighbor Table

Direct Sequence Spread Spectrum

Enhanced Connected Low Interference Channel Assignment Algorithm

Enhanced Topology- Controlled Interference- Aware Channel Assignment Algorithm

Frequency Hopping Spread Spectrum

Jain's Fairness Index

Fairness ratio

Gateway

Interference Range

Joint Resource and Channel Assignment

Least Interfering Channel

Local Minimum Spanning Tree

Medium Access Control

Mesh Client

Merge Based Channel Assignment for Interference Reduction

Maxflow based Channel Assignment and Routing

Multi- Channel MAC 


$\begin{array}{ll}\text { MPNT } & \text { Maximum Power Neighbor Table } \\ \text { MR } & \text { Mesh Router } \\ \text { MRMC } & \text { Multi Radio Multi Channel } \\ \text { MST } & \text { Minimum Spanning Tree } \\ \text { NAV } & \text { Network Allocation Vector } \\ \text { OFDM } & \text { Orthogonal Frequency Division Multiplexing } \\ \text { PDR } & \text { Packet Delivery Ratio } \\ \text { PCF } & \text { Point Coordination Function } \\ \text { PHY } & \text { Physical } \\ \text { RT } & \text { Random Topology } \\ \text { RTS } & \text { Request To Send } \\ \text { SPT } & \text { Shortest Path Tree } \\ \text { SRSC } & \text { Single Radio Single Channel } \\ \text { TCA } & \text { Topology Control Algorithm } \\ \text { TICA } & \text { Topology-Controlled Interference-Aware Channel Assignment } \\ \text { TR } & \text { Throughput Ratio } \\ \text { TR } & \text { Transmission Range } \\ \text { WMN } & \text { Wireless Mesh Network } \\ \text { UDP } & \text { User Datagram Protocol }\end{array}$




\section{Chapter - 1 : Introduction}

\subsection{Introduction}

This chapter discusses wireless mesh networks and their characteristics. It also explains the main motivation of the thesis and its contributions. Finally, it outlines the organization of the thesis.

\subsection{Wireless Mesh Networks}

Wireless Mesh Networks (WMNs) have recently emerged as a flexible, reliable and cost effective way of providing broadband internet access over large areas through multi hop communication.

A WMN is a special type of ad hoc network. WMNs are decentralized networks that are self configuring, self healing, reliable and scalable. Each node in a WMN may be a sender, receiver or may route packets for other nodes.

WMNs help overcome the drawback of Wireless Local Area Network (WLAN) architecture which is based on Digital Subscriber Line (DSL) where the last hop is wireless. They can be specifically useful and cost effective for community and neighborhood networks, broadband home networking, building automation, public safety networks etc.

A Wireless Mesh Network consists of three basic entities:

1. Mesh Clients (MCs): These are stationary or mobile nodes. They can be endpoint wireless users and can route packets on behalf of other nodes which may not be in direct transmission range of their destination.

2. Mesh Routers (MRs): These are access points that form a multi hop connection between the Mesh clients and the Gateway.

3. Gateways $(\mathbf{G W s})$ : These are access points that are connected to the wired network. 


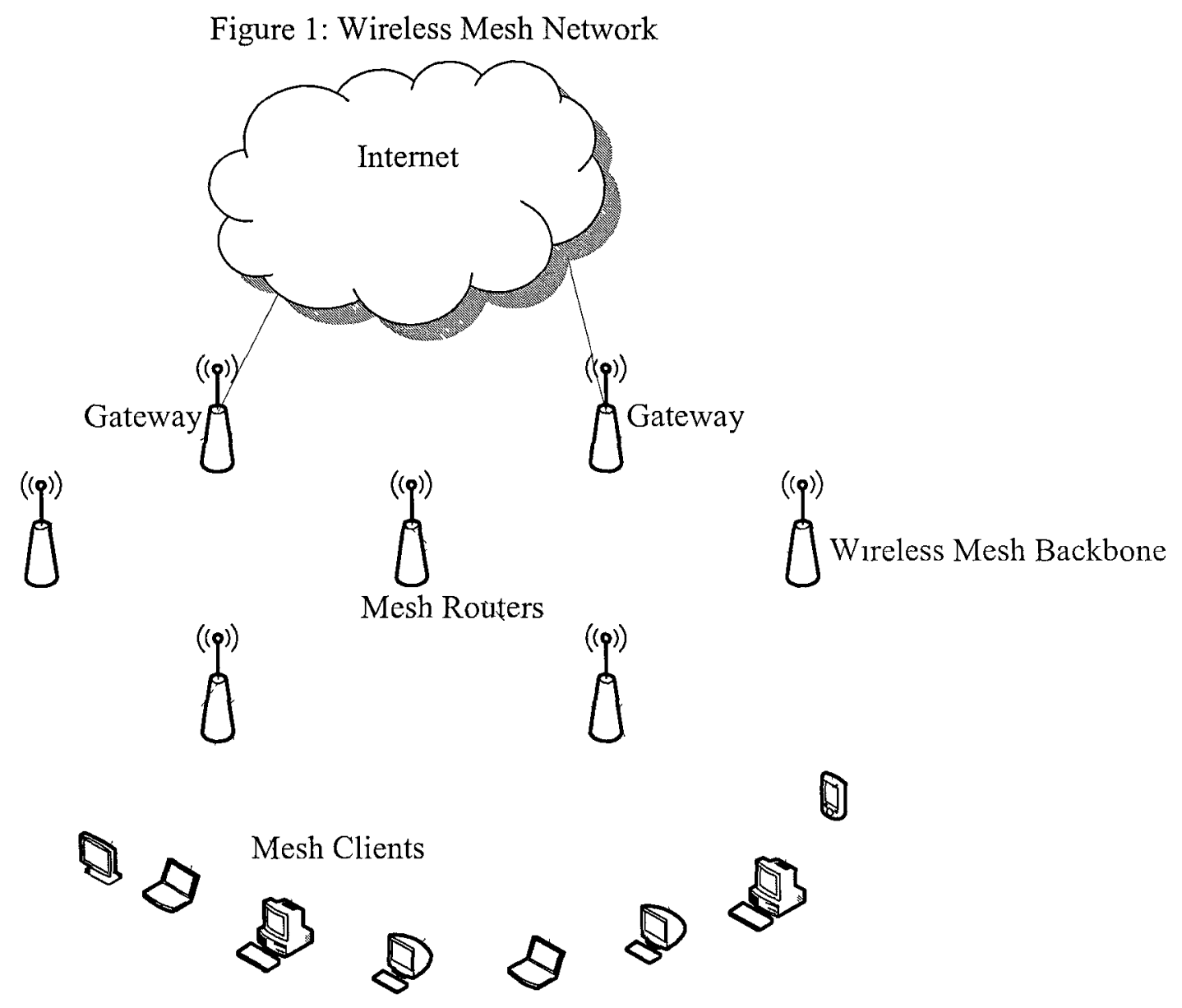

Some important characteristics of a WMN are:

1. Self healing and reliable: WMN are self healing. Each node in a WMN relays data packets of other nodes to their neighbors or to the access point in order to reach their final destination. In the event of a failure in one route, the packets are forwarded through an alternate route towards their destination. Thus, the self healng property makes WMNs reliable.

2. Self configuring: Network administration is minimal because the nodes are configured to learn about therr neighboring nodes and optimal routes to other nodes There is no need of a central controller.

3. Scalability: WMNs are scalable. The nodes should be software compatible with the other nodes in the network. However, increasing the number of nodes would imply reusing channels and packet collisions due to high traffic. This would lead to a drop 
in throughput. Hence, an important point to be kept in mind is how many nodes can be added before the throughput drops to an unacceptable level. Also, multiple gateways might need to be deployed so that the congestion is distributed among the gateways.

4. Economics: The above mentioned advantages lead to WMN being an economically viable option. The elimination of a central controller leads to lower administrative costs. Similarly, the self healing property of WMN reduces the cost of maintenance.

\subsection{Motivation}

The capacity of Single-Radio Single-Channel (SRSC) WMNs is limited due to collisions caused by hidden and exposed terminals. This is due to the interference caused by neighboring nodes which compete for the same channel [2]. Recent approaches aimed at improving the capacity of WMNs include Multi Radio Multi Channel (MRMC) WMNs, directional antennas and MIMO techniques.

A promising approach to alleviate the capacity problem is to equip each mesh node with multiple radios and assign orthogonal channels to the mesh nodes, hence facilitating simultaneous transmission and reception for neighboring nodes. This would result in efficient spectrum utilization and increased bandwidth for the network.

Although MRMC WMNs are emerging as a promising approach to overcome the capacity problem in SRSC WMNs, the number of available orthogonal channels is limited. In order to achieve their potential, MRMC WMNs must utilize an efficient channel assignment scheme which can intelligently allocate channels to radios. The key objective of a channel assignment scheme is to ensure network connectivity as well as minimize interference between mesh nodes through efficient reuse of the available channels [4].

\subsection{Contributions}

The main contribution of this thesis is an enhancement to a recently proposed channel assignment algorithm (CAA) called Topology-controlled Interference-aware Channel-assignment Algorithm (TICA) [5].

The important enhancements made to TICA are the following: 
1. AN ACCURATE CHANNEL ASSIGNMENT: The 2-way interference range edge coloring model, introduced in this thesis, leads to a better channel assignment strategy which helps in reducing conflicting channel assignments in most cases.

2. REDUCTION IN INTERFERENCE: Employing a Minimum Spanning Tree (MST) rooted at the gateway for power control. Utilizing MST for power control subsequently leads to minimal co-channel interference thereby increasing spatial channel reuse and reducing the occurrence of conflicting channels.

3. REDUCTION IN CONGESTION: In addition to this, to fully utilize the maximum possible radios out of the four radios of the gateway, the proposed algorithm is forced to build an MST from the GW utilizing its nearest neighbors.

\subsection{Thesis Organization}

The rest of the thesis is organized as follows. Chapter 2 presents existing literature related to channel assignment schemes, topology control mechanisms and fairness in wireless mesh networks. In Chapter 3, we present our problem statement. Chapter 4 discusses the problem of one-way interference range edge coloring associated with TICA and presents the all three channel assignment algorithms. Chapter 5 evaluates the performance of the proposed CAAs using simulation. Chapter 6 concludes the thesis along with some future research directions. 


\section{Chapter - 2 : Background and Related Literature}

\subsection{Introduction}

This chapter gives an overview of some IEEE $802.11 \mathrm{x}$ protocols and briefly discusses the physical (PHY) and medium access control (MAC) layer functionalities associated with them.

Related work on channel assignment schemes for MRMC WMNs is presented along with literature on topology control mechanisms and fairness in wireless networks.

\subsection{IEEE 802.11x Protocols}

IEEE $802.11 \mathrm{x}$ are a set of protocols for WLANs developed by a working group of IEEE. These protocols define PHY layer transmission technologies and the MAC layer functionalities [10]. Some of these protocols are discussed as follows:

\subsubsection{IEEE 802.11}

This was the first standard developed by the IEEE. It defines the use of three PHY layers for wireless communication namely Infrared, Frequency-Hopping Spread Spectrum (FHSS) and Direct Sequence Spread Spectrum (DSSS).

Under FHSS, a station transmits for a small interval of time at one frequency and then hops to a different frequency to continue the transmission. The frequency hopping pattern is known to all stations. Originally, this scheme was suggested to the USA as a security mechanism during World War II.

In DSSS, a spreading code is applied to each bit to spread the transmission. This method was also initially used as a security mechanism.

802.11 defines operations in the $2.4 \mathrm{GHz}$ band and has data rates of $1 \mathrm{Mbps}$ and 2 Mbps. Hence, two extensions to 802.11 were developed with an aim to increase the data rate. These were the $802.11 \mathrm{a}$ and $802.11 \mathrm{~b}$ standards [7]. 


\subsubsection{IEEE 802.11a}

This standard defines a new PHY layer that utilizes Orthogonal Frequency Division Multiplexing (OFDM). It has 12 non-overlapping channels. Theoretically, this can achieve a data rate of $54 \mathrm{Mbps}$ though practically, the achievable data rate is around 24 Mbps.

This standard defines operations in the $5 \mathrm{GHz}$ band. Since high frequencies attenuate faster than low frequencies, the range of stations operating on IEEE 802.11a is lower than those operating on 802.11 .

\subsubsection{IEEE 802.11b}

The IEEE $802.1 \mathrm{lb}$ standard is popularly known as Wi-Fi and uses DSSS as the PHY layers transmission technology. It provides data rate of $11 \mathrm{Mbps}$ and $5.5 \mathrm{Mbps}$. It operates in the $2.4 \mathrm{GHz}$ band, hence is backward compatible with 802.11 DSSS equipment and has a longer range than $802.1 \mathrm{la}$.

\subsubsection{IEEE 802.11g}

IEEE $802.11 \mathrm{~g}$ was developed with an aim to combine the advantages associated with IEEE 802.11a and IEEE 802.11b namely the high data rate of the former and the long range of the latter.

It uses OFDM as the PHY layer transmission technology and works in the 2.4 $\mathrm{GHz}$ band and provides a data rate of $54 \mathrm{Mbps}$

It also supports DSSS operations at 11, 5.5, 2 and $1 \mathrm{Mbps}$. Hence, IEEE 802.11g is backward compatible with 802.11 and $802.11 \mathrm{~b}$

\subsection{Medium Access Control (MAC) Layer}

The IEEE 802.11 MAC sub-layer defines two access methods: The Point Coordination Function (PCF) and The Distributed Coordination Function (DCF).

\subsubsection{PCF}

The PCF provides service without contention. In the PCF, the Access Point (AP) acts as the coordinator and allows stations in a cyclic manner to access the channel and 
transmit their data. Since APs are needed to ensure contention free service, PCF is restricted to infrastructure based networks.

\subsubsection{DCF}

DCF is the basic access scheme and utilizes the Carrier Sense Multiple Access with Collision Avoidance (CSMA/CA) mechanism. Here, stations have to contend for access to the channel and there is no central controller.

CSMA/CA uses two types of carrier sense: mandatory Physical Carrier Sense that monitors the signal strength of the channel, and optional Virtual Carrier Sense that uses the Request-To-Send/Clear-To-Send (RTS/CTS) handshake to reserve the medium prior to transmission.

\subsubsection{A Physical Carrier Sense}

Whenever a node has data to send, it senses the channel prior to transmitting the packet. If it senses signals that are greater than its carrier sense threshold it will defer from transmitting for a random interval of time. However, if the medium is free for a specific period of time called the Distributed Inter Frame Space (DIFS), the node will proceed with its transmission. Each node in a network has three basic ranges related to packet transmission:

Transmission Range (TR) is the range inside which a node can receive and decode packets correctly.

Interference Range (IR) is the range inside which any new transmission will interfere with packet reception

Carrier Sensing Range (CS) is the range within which a node can sense the signal and will defer from transmission

Usually, carrier sensing range and interference range are taken to be equal to each other $(C S=I R)$ and transmission range has to be less than or equal to carrier sensing range $(\mathrm{TR} \leq \mathrm{CS})$

If the CS range is too low (in other words, carrier sensing threshold is high), collisions will increase and if it is too high (or, carrier sensing threshold is low), the node will defer from transmission. Both cases will decrease throughput of the network. Since 
the carrier sensing range determines whether or not a node will go ahead with its transmission, it becomes imperative to set an appropriate value for it.

Issues with CSMA/CA:

Hidden Node Problem: There are three nodes A, B, C. Nodes A and B are within transmission range of each other and Node $\mathrm{C}$ and $\mathrm{B}$ are within transmission range of each other (Fig 2). Nodes $\mathrm{A}$ and $\mathrm{C}$ are not within transmission range and cannot detect each other's transmissions. Hence, transmissions by Nodes $\mathrm{A}$ and $\mathrm{C}$ can collide at Node B. This is the hidden node problem.

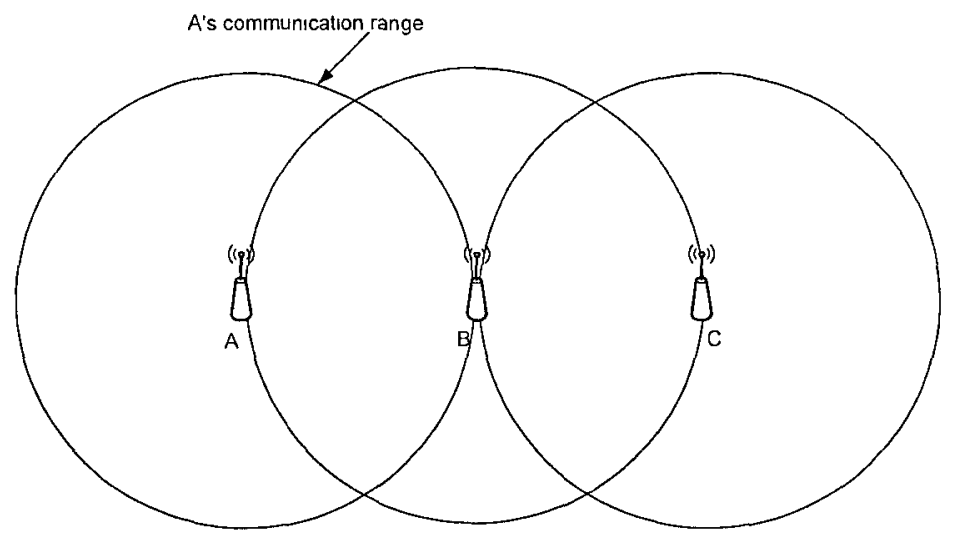

Figure 2: Hidden Node

Exposed Node problem: In Fig 3, Node B is transmitting to Node A and Node D wants to transmit to Node $C$. The transmissions can go on simultaneously without any collisions because Node $\mathrm{A}$ and $\mathrm{C}$ are not in transmission range but since Nodes $\mathrm{B}$ and $\mathrm{D}$ are within transmission range of each other, Node D defers transmission after carrier sensing the transmission of $B$.

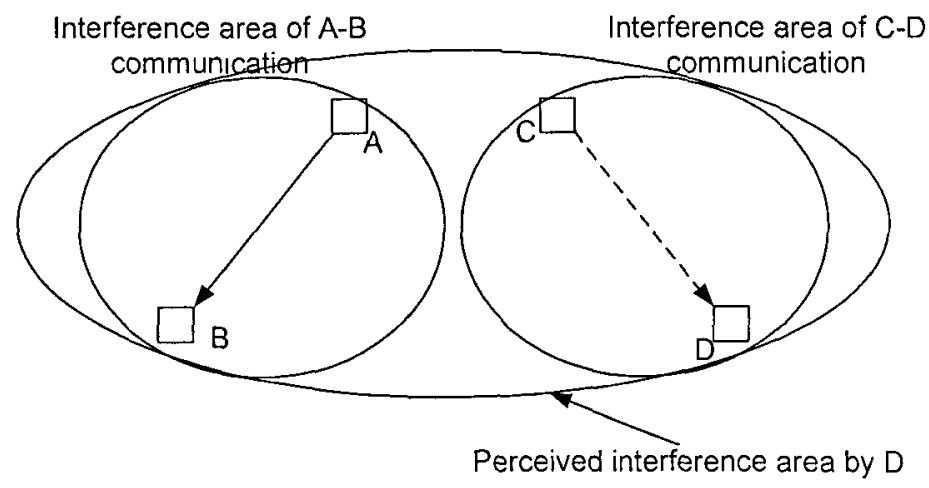

Figure 3: Exposed Node 


\subsubsection{B Virtual Carrier Sense}

The hidden and exposed node problems can be solved to a certain extent by the use of Virtual Carrier Sensing (VCS). In this scheme, the node which has data to transmit sends an RTS (Request to send) to the intended receiver. If it receives a CTS (Clear to Send) message, it goes on with the transmission and all neighboring nodes which hear the RTS or CTS or both, will defer from transmitting. The RTS and CTS have information about how long the transmission will last and all over-hearing nodes update their NAV (Network Allocation Vector) and refrain from transmitting for that time. After the transmission is complete, all nodes that were waiting would start attempting the channel simultaneously. To overcome this problem, each node has a back off timer which is initialized to a random back off value and starts decrementing after the transmission is complete. Nodes may start transmitting only once the back off timer has reached zero. Since the probability of more than one node having the same back-off value is very low, chances of nodes attempting to access the channel simultaneously are very low too.

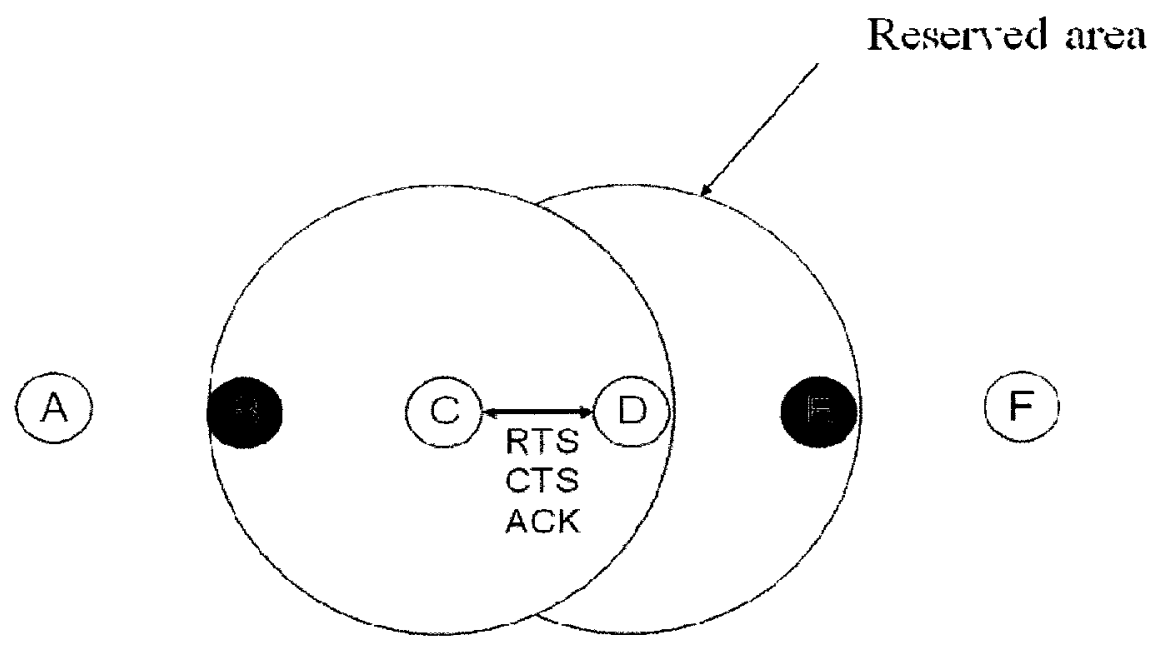

Figure 4: Virtual Carrier Sense 


\subsection{Related Literature}

\subsubsection{Channel Assignment Schemes}

Channel Assignment in a WMN is an important task for optimal utilization of available resources. Multi radio Multi channel WMNs have the potential to achieve much higher throughput because a node can have simultaneous transmissions and receptions on different radios. A channel assignment scheme has to keep the network connectivity because in order to communicate, two neighbors should have their radios on the same channel. However, reusing the same channel in a neighborhood must be minimized/ optimized because simultaneous transmissions on the same channel will lead to interference which will lead to a decrease in throughput.

Any channel allocation scheme needs to consider the following:

- Maintain network connectivity.

- Minimize interference between neighboring nodes.

- Provide adequate and optimal reuse of channels.

In a WMN, an Access Point (AP) that is connected to the wired network is called a Gateway $(\mathrm{GW})$; whereas APs without wired connections are called Mesh Routers (MRs). These MRs connect to the GW through one or more hops. In centralized CAAs, the gateway acts as the central controller and is responsible for allocating channels to the multi-radio MRs [8]. The following are some related centralized CAAs which have been proposed in the literature recently.

Multi Channel MAC (MMAC) is a Dynamic Channel Assignment Scheme. In this scheme, each node has only one transceiver but it can switch channels dynamically to reduce interference and improve network capacity [9]. When a node (A) wants to transmit data to another node (B), it sends its preferred channel list (PCL). Node B compares it to its own PCL and chooses a channel and sends information about it on an ACK back to node A. When node A gets this information, it sends another ACK with the channel information and both nodes start using the desired channel. All nodes that overhear this handshake defer their respective transmissions on the selected channel. 
In [11], the Joint Resource and Channel Assignment (JRCA) algorithm was introduced. This algorithm determines the number of radios required at each node based on the traffic demand and produces the channel assignment for each radio, such that the interference among the links operating on the same channel is minimized.

In [12], the Merge Based Channel Assignment for Interference Reduction (MCAIR) was proposed, which initially assigns channels by finding the degree of the conflict graph without considering any constraints for available channels or radios. It is assumed in [12] that if two links are using the same channel, these links will interfere with each other if they are within two hops of each other.

The Maxflow-based Channel Assignment and Routing (MCAR) algorithm presented in [13] splits the channel assignment into two stages. In the first stage, links are sorted into groups based on the flows they carry, while in the second stage, a channel is selected for each group and is assigned to all links of this group. If it is possible to do so, different channels are assigned to groups containing interfering links.

The Breadth First Search (BFS-CA) algorithm is introduced in [14]. It works on multi channel multi radio WMN and the objective of this algorithm is to minimize the interference between mesh routers as well as between the mesh network and other collocated wireless networks. This algorithm takes interference level estimate and multi radio conflict graph as an input. The interference level is used to decide the default channel (the one with the minimum interference level) and the multi conflict graph is used to find the non-default radios. Each node has a default radio which operates on a common channel throughout the network thus maintaining common connectivity and at the same time fallback routes.

Though all of these CAAs are interference-aware, unlike e-TICA, they do not employ the technique of two-way interference range edge coloring.

\subsubsection{Topology Control Schemes}

Topology control in WMNs is typically targeted towards reducing interference and improving spectral efficiency while maintaining network connectivity. Interference is confined by lowering the transmit power. Therefore, link length is taken as a metric to 
control interference. Since transmit power is directly proportional to the distance between the nodes, a reasonable strategy is to replace the long links with shorter ones.

In [16], the network topology has been built using MST and channel assignment has been done through the centralized tabu search. The motivation for using MST in [16] is that shorter links imply more capacity in WMN and also reduce interference with nearby links which use the same channel.

In [17], a CAA called Connected Low Interference Channel Assignment (CLICA) is presented which randomly selects a node and from that node, traverses other nodes in depth-first order assigning channels to minimize interference.

In [18], Enhanced Connected Low Interference Channel Assignment (ECLICA) and Minimum Spanning Tree Channel Assignment (MSTCA) algorithm has been proposed. ECLICA is a modified version of CLICA and utilizes the idle radios left by the CLICA. In MSTCA, an MST is derived from the given network topology in order to find links carrying the minimum traffic load. The authors have shown that the minimum spanning tree approach results in minimal co-channel interference and hence minimal aggregate network interference.

Local Minimum Spanning Tree (LMST) is a TCA for multi hop wireless networks presented in [19]. LMST uses MST to achieve short link lengths resulting in a smaller transmission power needed to maintain connectivity and a smaller average node degree resulting in fewer neighbors and hence reduced medium access contention.

\subsubsection{Fairness in wireless networks}

Since the main network resource, namely the spectrum is limited; it must be shared fairly among the contending nodes. Achieving fairness in WMNs can be categorized in terms of per-node and per-flow fairness. Per-flow faimess refers to equal share of the data among flows arriving at the gateway. Unfairness among flows arises due to multiple flows sharing the same link. This causes congestion at such links which leads to unfairness among flows reaching the gateway. Per-node fairness refers to equal access for each node to the wireless medium. Unfairness in medium access arises in MRMC WMNs due to some nodes operating on a conflicting channel and contending with each 
other for channel access. Single-radio single-channel WMNs experience unfairness due to hidden terminal problem in CSMA/CA based wireless networks [20], [21].

In [20], the authors have attributed unfairness in ad-hoc networks to the Binary Exponential Back-off algorithm used in CSMA/CA networks for adjusting the maximum contention window size based on the number of contending nodes. They have concluded that the larger the contention window size, the lower the scheduling rate for the node and have proposed distributed scheduling methods for providing fairness.

The authors have proposed an algorithm in [22] to improve the fairness by differentiating the traffic among the connections in a wireless multi-hop network.

The Max-Chop algorithm in [23] utilizes channel hopping to improve fairness in multi-hop wireless infrastructure networks. Here, all nodes use all channels to uniformly divide the bandwidth. In [24], the authors propose a receiving node assistance feature in addition to the existing CSMA/CA protocol to remove exposed terminal problem and enhance fairness in multi-hop wireless networks.

From the related work, we conclude that shorter hops achieved through MST help in curtailing the interfering environment. They also provide medium access fairness by eliminating the conflicting channels thereby removing contention among nodes to access the channel. Our proposed CAA, e-TICA2 for MRMC WMNs, is therefore based on this approach and the same has been verified by the simulation results.

In a random topology wireless mesh network, asymmetric lengths of links results in different interference ranges of nodes. Hence, finding an appropriate channel from the available eleven channels is crucial to the performance of the algorithm because reusing a channel within the interference range of either node of a link will lead to degradation of network performance.

\subsection{TICA}

Topology controlled Interference aware Channel assignment Algorithm (TICA) is a centralized and fixed CAA which uses topology control to build connectivity between nodes and applies power control between nodes to reduce interference and to increase spatial reuse. TICA runs on the 802.11a standard which defines an OFDM PHY layer which has 12 non-overlapping channels. In a wireless mesh network, the mesh nodes are 
access points which act as sources and relay information wirelessly to and from the gateway. The gateway is the only node that has a wired connection to the internet. The mesh nodes form a wireless backbone. Hence, all traffic flows to and from the gateway. In this thesis, regardless of its position, node 15 is taken as the gateway.

The operation of TICA can be divided into the following phases:

1. Topology Control Phase

2. Connectivity Phase

3. Building the Minimum Power based Tree

4. Channel Assignment Phase

These different phases of TICA are described in detail in the next chapter.

\subsection{Interference Modeling}

A common approach used by CAAs for interference modeling is the distance-2 edge coloring [6]. Two edges of a graph $\mathrm{G}$ are within distance-2 of each other if either they are adjacent, or there is some other edge that is adjacent to both of them. Distance-2 edge coloring of $\mathrm{G}$ is an assignment of colors (channels) to edges such that any two edges within distance- 2 of each other receive different colors. This means that edges that are adjacent or have a common edge between them as shown in Figure 5 should not be assigned the same color. It has been shown in [6] that for $K \geq 4$, where $K$ is the number of available colors, the distance- 2 edge coloring problem, also known as strong edge coloring problem, is NP-complete.
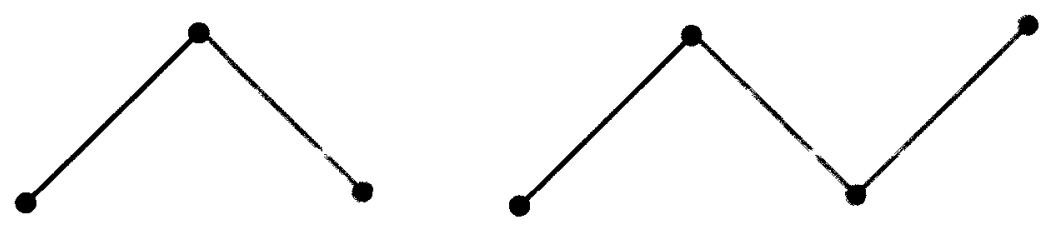

Figure 5: Distance-2 edge coloring

To minimize co-channel interference in a $\mathrm{WMN}$, it is necessary to assign channels to links such that links within the interference range of each other are assigned different channels. This problem has been termed as interference-range edge coloring and was introduced in TICA [5]. In a grid topology where links are of equal length, the 
interference-range edge coloring is identical to distance-2 edge coloring. However, in a random topology with asymmetric links, it can be more complex than distance- 2 edge coloring. 


\section{Chapter - 3 : Problem Statement}

\subsection{Introduction}

The capacity of SRSC WMNs is limited due to collisions caused by hidden and exposed terminals. This is due to the interference caused by adjacent mesh nodes which transmit and receive on the same channel. This problem can be alleviated by equipping each mesh node with multiple radios, and assigning orthogonal channels to the mesh nodes within the interference range. Although, MRMC WMNs can achieve improved performance due to the availability of multiple radios and multiple channels, the number of available orthogonal channels is limited. In order to achieve high capacity with limited number of orthogonal channels, we reduce the interference in the network by adjusting the transmit power of each mesh node. Then, we utilize an efficient channel assignment scheme similar to TICA. The objective of a channel assignment scheme is to ensure network connectivity as well as minimize the interference among mesh nodes.

\subsection{Issues with TICA}

TICA, which is a centralized and fixed CAA uses topology control to build a connectivity graph and then uses the Shortest Path Tree (SPT) approach to build a minimum power based tree with a maximum node degree of four. It applies power control at mesh nodes to reduce interference and increase spatial reuse. It uses interference-range edge coloring for assigning channels to links. In TICA, we use the following procedure:

1. For each new link, we determine the interference range of both nodes and list all the channels already assigned within that range.

2. We exclude the assigned channels from the list of channels and inspect the remaining channels.

3. If we have a channel that is not assigned within the interference range, we assign that channel to the link. 
4. Otherwise, we chose a channel that has the least amount of interference and assign it to the link.

However, the procedure outlined above could miss interference that affects the link in question as illustrated in Figure 6.

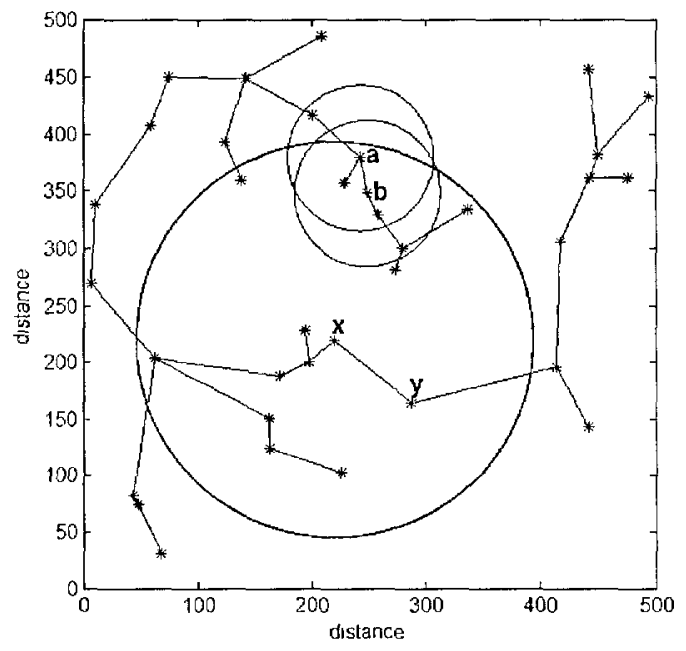

Figure 6: Hidden Link Problem

Specifically, if a link $a-b$, which has nodes $a$ and $b$ as its end nodes, lies in the interference range of node $x$ which is an end node of a link $x-y$, nodes $x$ and $y$ may not lie in the interference range of nodes $a$ and $b$. Hence, link $x-y$ becomes hidden during the channel assignment (CA) of link $a$ - $b$, which is called the hidden link problem in this thesis. The interference-range edge coloring approach used by TICA suffers from this problem, and this approach is referred to as one-way interference-range edge coloring. Links $a-b$ and $x-y$, which are within the interference range of their constituent nodes, could be assigned the same channel in one-way interference-range edge coloring due to the hidden link problem, which would cause co-channel interference and may result in decreased network throughput and fairness.

\subsection{Problem Statement: e-TICA and e-TICA2}

\subsection{1 e-TICA}

In this thesis, the hidden link problem is addressed by proposing an improved algorithm based on TICA, called enhanced-TICA (e-TICA). 
e-TICA utilizes two-way interference-range edge coloring for channel assignment whereby it inspects the interference range of both nodes associated with the link to which a channel is to be assigned. Additionally, the algorithm also inspects the channels assigned to the links within the interference range of which these two nodes lie. Specifically, for the scenario shown in Figure 6, to assign a channel to link $a-b$, e-TICA will inspect the interference range of nodes $a$ and $b$ and also the channel assigned to link $x-y$. This removes the hidden link problem and leads to a more accurate channel assignment algorithm.

\section{3 .2 e-TICA2}

TICA and e-TICA employ the shortest path tree approach to build a minimum power based tree from the GW to each node in the network. Here the weight of each link is the minimum transmit power required by a node to reach its neighbor. Hence, the shortest path from the gateway to each node indicates the minimum total power needed to reach each node from the gateway. This approach works well in most random topologies. However, in some random topologies, the presence of long links leads to conflicting channels which results in co-channel interference. This is explained in greater detail in the next chapter in section 4.4.1.

Since the long links contribute to interference, they should be replaced with shorter links wherever possible. Based on this concept, in this thesis, a modified CAA $e$ TICA2 is introduced which employs a minimum spanning tree rooted at the GW instead of an SPT as used in TICA and e-TICA. The MST approach leads to a reduction in conflicting channels and improves network throughput. MST leads to more but smaller hops instead of few but longer hops in SPT. Hence, utilizing MST for building the minimum power-based tree should lead to minimal co-channel interference thereby increasing spatial channel reuse. 


\section{Chapter - 4 : Description of the Proposed}

\section{Algorithms}

\subsection{Introduction}

Since all our work is based on TICA, we include a description of the TICA scheme in the next section. e-TICA has been proposed as an improvement over TICA. It uses two-way interference range edge coloring for channel assignment. This algorithm results in an improved channel assignment which leads to an enhancement in the network throughput.

e-TICA is further improvised by replacing the shortest path approach for building a minimum power based tree by employing a MST rooted at the gateway. The gateway is also forced to use its nearest neighbors while building the MST so that the load is divided among the radios of the gateway and congestion is reduced. This modified algorithm is referred to as e-TICA2.

In all three schemes proposed here, each MR is equipped with five radios which operate on IEEE 802.11 a channels ( $5 \mathrm{GHz}$ band). One of these radios is used for control traffic while the other radios are used for data traffic. Out of the 12 available nonoverlapping 802.11a channels, channel number 12 is used for the control radio on each MR and the remaining 11 channels are used for data radios. Since each MR is equipped with 4 data radios, it can communicate with a maximum of four transmission range neighbors simultaneously using these radios. This implies a maximum node degree of four per node. 


\subsection{TICA}

The operations of TICA, which is a centralized and fixed CAA and uses topology control to build connectivity and applies power control between nodes to reduce interference, can be divided into the following phases:

1. Topology Control Phase

2. Connectivity Phase

3. Building the Minimum Power based Tree

4. Channel Assignment Phase

\subsubsection{Topology control phase}

The network comprises of $36 \mathrm{MRs}$ also referred to as nodes and irrespective of its location, node 15 is chosen to be the gateway for each topology.

Initially, all nodes transmit at their maximum power and build a table of all neighbors that are located in this range. Each node sends its location information and its maximum power neighbor table (MPNT) to the gateway. Consider random topology 3 as shown in Figure 7. The circular disk in the figure indicates the maximum transmission range of node 1 .

Table 1 shows all the nodes that are within the maximum transmission range of node 1 .

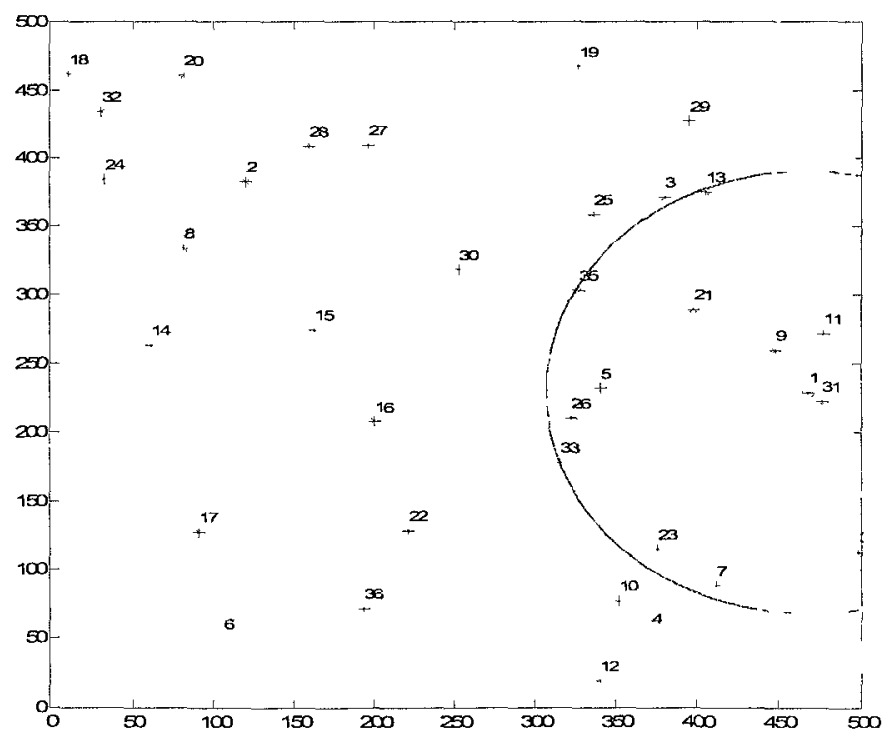

Figure 7: Maximum Power Transmission Range of Node 1 
Table 1: Maximum Power Transmission Range neighbors of Node 1

\begin{tabular}{|l|l|}
\hline $\begin{array}{l}\text { Maximum Transmission Range Neighbors } \\
\text { of Node } 1\end{array}$ & Distance between Node 1 and neighbor (m) \\
\hline 31 & 11 \\
\hline 9 & 36 \\
\hline 11 & 43 \\
\hline 21 & 92 \\
\hline 34 & 120 \\
\hline 5 & 127 \\
\hline 23 & 146 \\
\hline 26 & 147 \\
\hline 7 & 152 \\
\hline 13 & 159 \\
\hline 35 & 159 \\
\hline 33 & 160 \\
\hline
\end{tabular}

\subsubsection{Connectivity phase}

The GW has information about each node's location and its maximum transmission range neighbors. It selects the nearest neighbors for each node in the network using the 'select $x$ for less than $x$ ' topology control algorithm (TCA). The GW initially executes 'select one for less than one' and finds the closest neighbor for each node making sure that each node must have at least one neighbor. It then checks for network connectivity. If the network is connected, it moves on to the next phase, which is building the shortest path tree from each end node to the GW, otherwise, it executes 'select two for less than two' algorithm and checks for connectivity. This process is repeated using 'select three for less than three' and 'select four for less than four' until the network is fully connected.

Table 2 shows the direct neighbor table (DNT) for node 1 . Note that only four neighbors from the MPNT are left with node 1 as the other nodes are closer to some other 
node. For instance, node 21 is closer to nodes 5, 9 and 35, than it is to node 1 , therefore, it will be removed from the DNT of node 1.

Figure 8 shows the connectivity graph for random topology 3 which results from the select 2 for less than 2 TCA. As can be seen, the topology is disconnected at nodes 22 and 23 and both of them have two neighbors each. The select 3 for less than 3 TCA results in a connected topology (Figure 9). It should be noted that this TCA increases the number of neighbors of nodes 22 and 23 from two to three, hence resulting in a connected topology.

Table 2: Direct Neighbor Table of Node 1

\begin{tabular}{|l|l|}
\hline Direct neighbors of Node 1 & Distance between Node 1 and neighbor $(\mathrm{m})$ \\
\hline 31 & 11 \\
\hline 9 & 36 \\
\hline 11 & 43 \\
\hline 34 & 120 \\
\hline
\end{tabular}




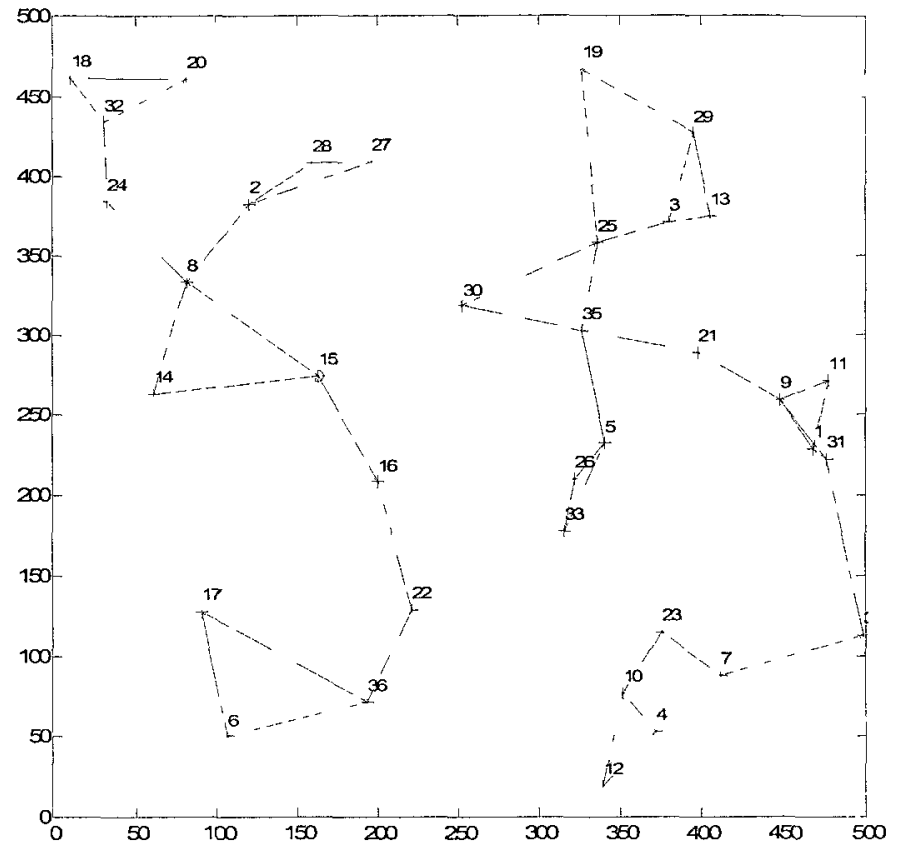

Figure 8: Connectivity Graph 'select 2 for less than 2' TCA

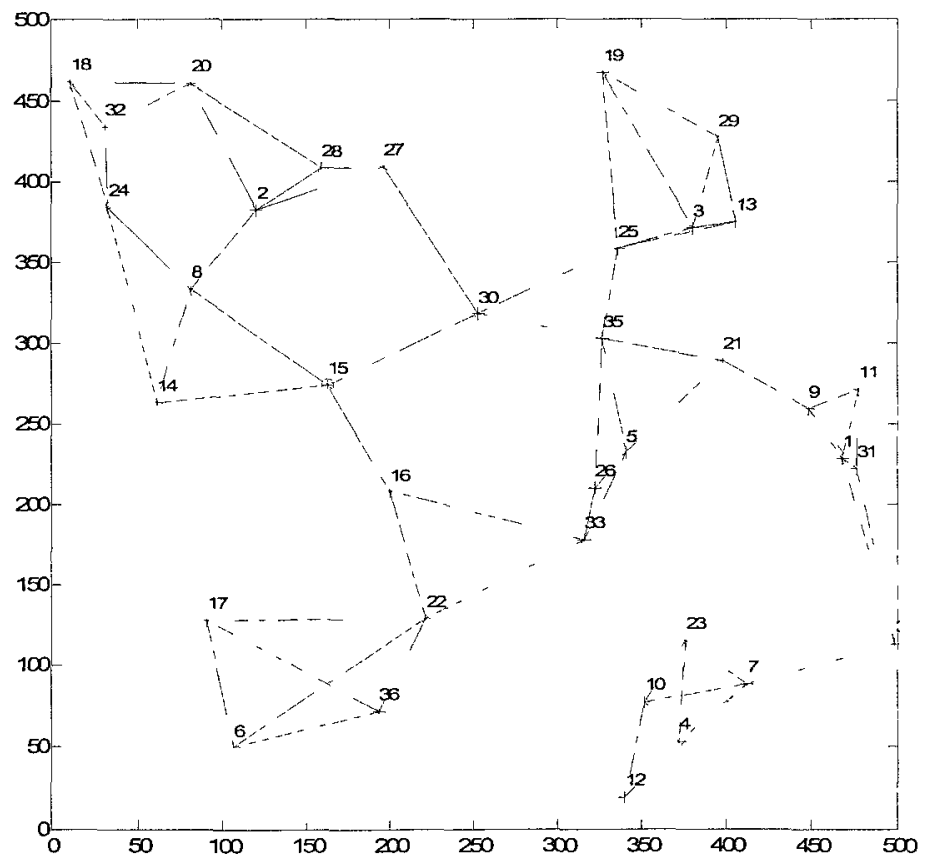

Figure 9: Connectivity Graph 'select 3 for less than 3' TCA 


\subsubsection{Minimum Power Shortest Path Tree}

In this phase, the GW builds a shortest path tree with a maximum node degree of four, from itself to each end node (see Figure 10) using the links in the connectivity graph. The weight of each link is the transmit power used by a node to reach its next hop neighbor. Hence, the shortest path from each end node to the gateway represents the minimum power route from the gateway to each node.

If the distance between two nodes is less than the cross-over distance, Free Space propagation model is used otherwise, Two-ray propagation model is used. Cross-over distance is given by

$$
\text { Cross_over_dist }=\frac{4 \pi h h_{r}}{\lambda} \text {, }
$$

where $h_{t}$ and $h r$ are the antenna heights of the transmitter and receiver, respectively. The minimum power for the free-space propagation model is calculated as

$$
P_{m n}=\frac{R x \operatorname{Thresh}(4 \pi d)^{2}}{G_{t} G, \lambda^{2}} .
$$

The minimum power for the two-ray propagation model is given by

$$
P_{\mathrm{min}}=\frac{R x T h r e s h(d)^{4}}{G_{i} G_{r} h_{t}^{2} h_{i}^{2}}
$$

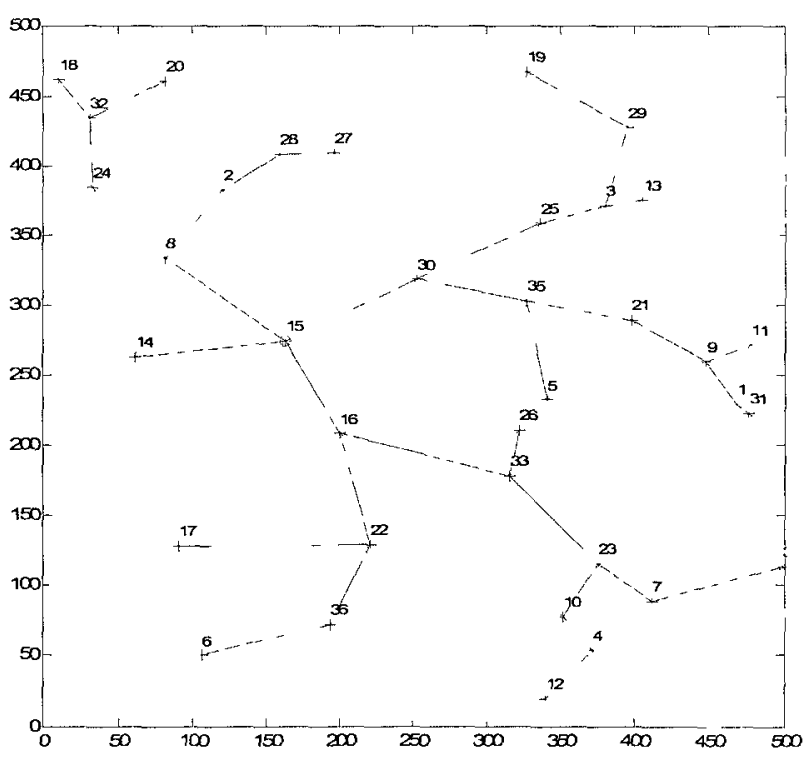

Figure 10: Shortest Path Tree from GW (Node 15) to all other nodes 


\subsubsection{Channel Assignment phase}

Channel assignment is also performed at the gateway. Link ranking is done during the channel assignment phase and links are ranked in decreasing order of the number of nodes that use those links to reach the gateway. Links that are ranked higher are given priority for channel assignment. Consequently, the links which are closer to the gateway are used by a greater number of nodes and hence are allocated a higher rank. For instance, link $15-16$ is ranked the highest as it is used by 13 nodes to reach the GW. If two links have the same number of nodes using them, a second ranking is done based on the power needed by the end node of the link to reach the GW. For example, as shown in Table 3, links 15-16 and 15-30 both have 13 nodes using them to reach the GW, however the power needed by link $15-16$ is lower than the power needed by link 15-30. Therefore, link 15-16 is ranked higher than link 15-30.

There are twelve orthogonal channels available for use in the 802.11a standard. Out of these, eleven are used for data and the twelfth is used as a control channel on which topology and channel assignment messages are exchanged between the nodes and the GW. The channel assignment algorithm assigns the eleven orthogonal channels to the eleven highest ranked links. This is done because the eleven highest ranked links are the ones which are used the most and hence, are given a higher priority. To assign a channel to the twelfth link, the algorithm checks the interference range of the first node of the link. TICA assumes the interference range to be twice the transmission range. The channels used within the interference range of the first node cannot be reused. Similarly, the interference range of the second node of the link is also inspected. All the channels that have been used in this interference range can also not be reused. After inspecting the interference ranges of both the nodes that form the link, the algorithm checks if there is any available channel that has not been used within the interference range of both the nodes. Such a channel is called a Non-Conflicting Channel. If there are one or more non conflicting channels, the highest numbered channel is allocated to the link. If the algorithm cannot find a non conflicting channel, an LIC has to be reused. The algorithm computes an LIC based on the rank of the link it is already being used on, the number of links that are using that particular channel and the distance from the node. 
Table 3 shows the 13 highest ranked links for random topology 3 and the channels allocated to them.

Out of the eleven available channels, those that are not assigned to any link within the interference range of both nodes that constitute a link are termed as non-conflicting channels. If the gateway cannot find any such channel for the twelfth ranked link and onwards, it selects a channel that causes minimum interference to the link. Such a channel is called a Least Interfering Channel (LIC).

To assign a channel to the twelfth link, the algorithm checks the interference range of the first node of the link. TICA assumes the interference range to be twice the transmission range. The channels used within the interference range of the first node cannot be reused. Similarly, the interference range of the second node of the link is also inspected. All the channels that have been used in this interference range can also not be reused. After inspecting the interference ranges of both the nodes that form the link, the algorithm checks if there is any available channel that has not been used within the interference range of both the nodes. Such a channel is called a Non-Conflicting Channel. If there are one or more non conflicting channels, the highest numbered channel is allocated to the link. If the algorithm cannot find a non conflicting channel, an LIC has to be reused. The algorithm computes an LIC based on the rank of the link it is already being used on, the number of links that are using that particular channel and the distance from the node.

Table 3: Link Ranking and Channel Allocation

\begin{tabular}{|c|c|c|c|c|}
\hline \multicolumn{2}{|c|}{ Link } & Rank1 & Rank2 & Channel Allocated \\
\hline 15 & 16 & 13 & 108 & 1 \\
\hline 15 & 30 & 13 & 187 & 2 \\
\hline 15 & 8 & 8 & 187 & 3 \\
\hline 16 & 33 & 8 & 378 & 4 \\
\hline 30 & 35 & 7 & 295 & 5 \\
\hline 33 & 23 & 6 & 520 & 6 \\
\hline 30 & 25 & 5 & 346 & 7 \\
\hline 35 & 21 & 5 & 392 & 8 \\
\hline 16 & 22 & 4 & 282 & 9 \\
\hline 8 & 24 & 4 & 234 & 10 \\
\hline 25 & 3 & 4 & 455 & 11 \\
\hline 21 & 9 & 4 & 386 & 10 \\
\hline 8 & 2 & 3 & 262 & 11 \\
\hline
\end{tabular}


The interference level of each channel is calculated using the following formula:

$$
(I L)_{\imath}=\sum_{m}\left(\frac{r_{m}}{R}\right)\left(\frac{1}{d_{m}^{\alpha}}\right)
$$

Where $i$ is the channel, $r$ is the rank of the link using channel $i, R$ is the maximum rank assigned to the links in the tree, $m$ is a link using channel $i$ within the interference range of the nodes that constitute the twelfth ranked link, $\alpha$ is the path loss exponent ( 2 or 4 depending on the cross over distance) and $d$ is the distance between the node and the link.

In the case of random topology 3 , to assign a channel to link 21-9 which is the twelfth ranked link, the algorithm inspects the interference range of both nodes 21 and 9 . The circular disks in Figure 11 indicate the interference range of node 21 and 9 respectively. The channels that are included in this interference range are 5, 7,8 and 11 . Hence the algorithm does not choose any of these channels and allocates channel 10 to link 9-21. Channel 10 is chosen by the algorithm because it is being used by the 10th ranked link which is low in priority than the other 9 high ranked links.

The nodes are then informed of their one hop neighbors to the GW and the channel to be used for the link. The mesh node applies power control based on the distance between itself and its one hop neighbor. 


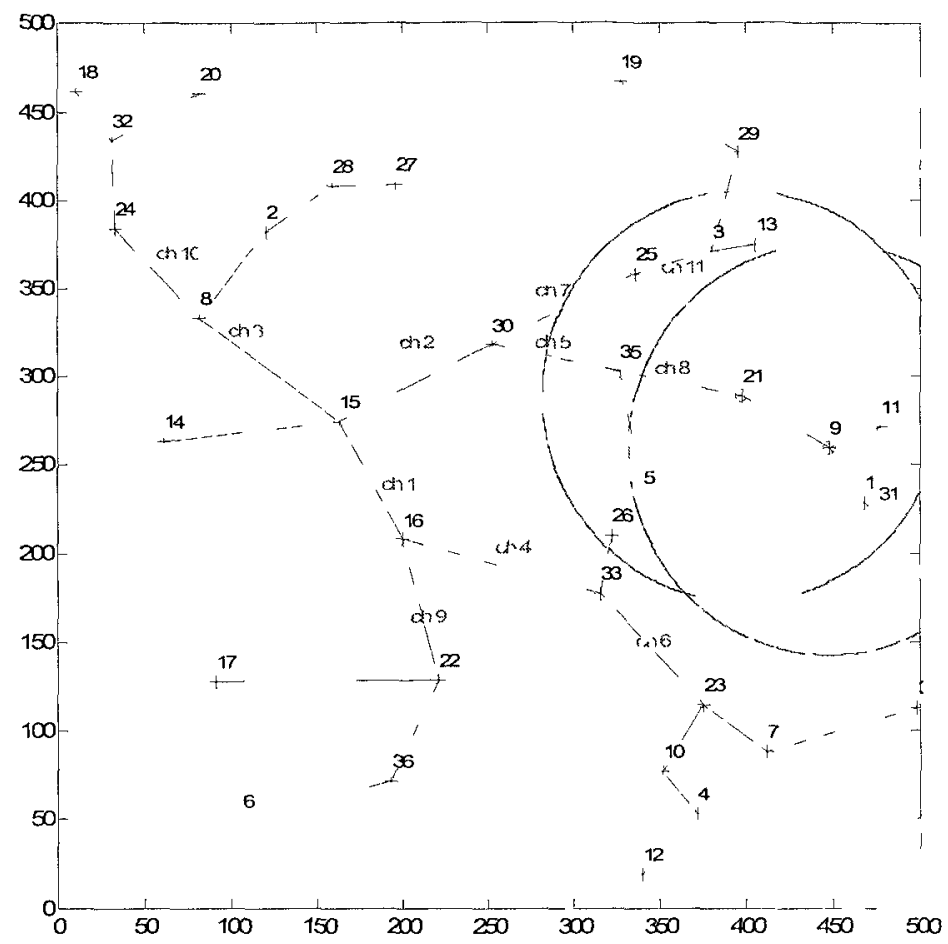

Figure 11: Interference range of node 21 and 9

The propagation model is chosen to be the two-ray propagation model if the distance between two nodes is greater than the cross over distance and the free space propagation model otherwise.

\section{3 e-TICA}

Like TICA, e-TICA uses topology control to build a connectivity graph and then uses the SPT approach to build a minimum power based tree with a maximum node degree of four. Links of the minimum power based SPT are ranked in decreasing order of the number of nodes that use those links to reach the gateway. During the channel assignment phase, links that are ranked higher are given priority. e-TICA begins the channel assignment by assigning the eleven non overlapping channels to the eleven highest-ranked links such that channel 1 is assigned to the highest ranked link. For the twelfth ranked link and onwards, it checks the channel assignment of all links within the interference range of both nodes that constitute that link. If the algorithm finds one or more non-conflicting channels, i.e. a channel that is not being used within the interference range of either node of the link, it assigns the highest numbered channel to 
the link. If the algorithm cannot find any non-conflicting channel, it selects an LIC for that link.

Unlike TICA, e-TICA uses 2-way interference-range edge coloring to identify LICs and to assign channels to links of the minimum power based SPT. The 2-way interference range edge coloring method is described in the next section.

\subsection{An accurate channel assignment algorithm}

Motivation: TICA uses interference range edge coloring for assigning a channel to a link, whereby it inspects the channel-assigned links within the interference range of both mesh nodes that constitute that link before assigning it a channel. However, this approach of one-way interference range edge coloring does not consider those links in whose interference range the nodes in consideration are located, which leads to conflicting channel assignments and decreased network throughput and fairness. This drawback of TICA has been addressed by employing two-way interference range edge coloring.

e-TICA: The channel assignment phase of TICA has been modified for the algorithm to yield better throughput and fairness. Random topology 8 has been investigated, as shown in Figure 12, where TICA has been used for channel assignment. This scenario consists of a random topology comprised of 36 MRs. During channel assignment, the interference range is assumed to be twice the transmission range and is indicated by the circular disk in Figure 12 and Figure 13. Link 23-19 is ranked high as it is used by 9 nodes to reach the gateway. Hence, it is allocated channel 9 which has not been allocated to any other channel yet. Link 1-17 has a lower rank as it is used by 4 nodes to reach the gateway and since the algorithm has already allocated the 11 channels, it searches the interference range of nodes 1 and 17 for an available channel. Link 1-17 is assigned channel 9 as the algorithm cannot find any other link using channel 9 , as shown in Figure 13. From Figure 12, it can be observed that nodes 1 and 17 are in the interference range of nodes 23 and 19 which are end nodes of the link 23-19. However, this is not identified by TICA because it is based on one-way interference range edge coloring. In other words, link 23-19 becomes a hidden link during the channel assignment phase of link 1-17. Eventually, links 1-17 and 23-19 share the same channel even though 
the nodes that constitute them are within the interference range of each other, which leads to a degradation in the throughput of the network.

The proposed algorithm, e-TICA, resolves the above problem by using two-way interference range edge coloring. When channels are being assigned to links, e-TICA inspects the links in the interference range of both nodes associated with that particular link, as well as the links in whose interference range these nodes are located. Specifically, in order to assign a channel to "ink 1-17, e-TICA checks the channels being used in the interference range of nodes 1 and 17 as well as the channel assigned to link 23-19. We term the new model as two-way interference range edge coloring, which implies that links formed by nodes which are within the interference range of each other will not be allocated the same channel, provided that there is a channel available for allocation. Table 4 summarizes the channel assignment with TICA as well as e-TICA for the scenario described above. As is evident from this table, e-TICA allocates channel 7 to link 1-17 instead of channel 9. TICA allocates the same channel to links 19-36 and 1-31 even though the nodes that constitute them are within the interference of each other whereas e-TICA allocates channel 8 to link 1-31 instead of channel 11, thereby eliminating the hidden link problem.

Table 4: Comparison of the channel assignment done by TICA and e-TICA

\begin{tabular}{|c|c|c|}
\hline Link & Channel Assignment TICA & $\begin{array}{c}\text { Channel Assignment } \\
\text { e- TICA }\end{array}$ \\
\hline $23-19$ & 9 & 9 \\
\hline $1-17$ & 9 & 7 \\
\hline $19-16$ & 11 & 11 \\
\hline $1-31$ & 11 & 8 \\
\hline
\end{tabular}




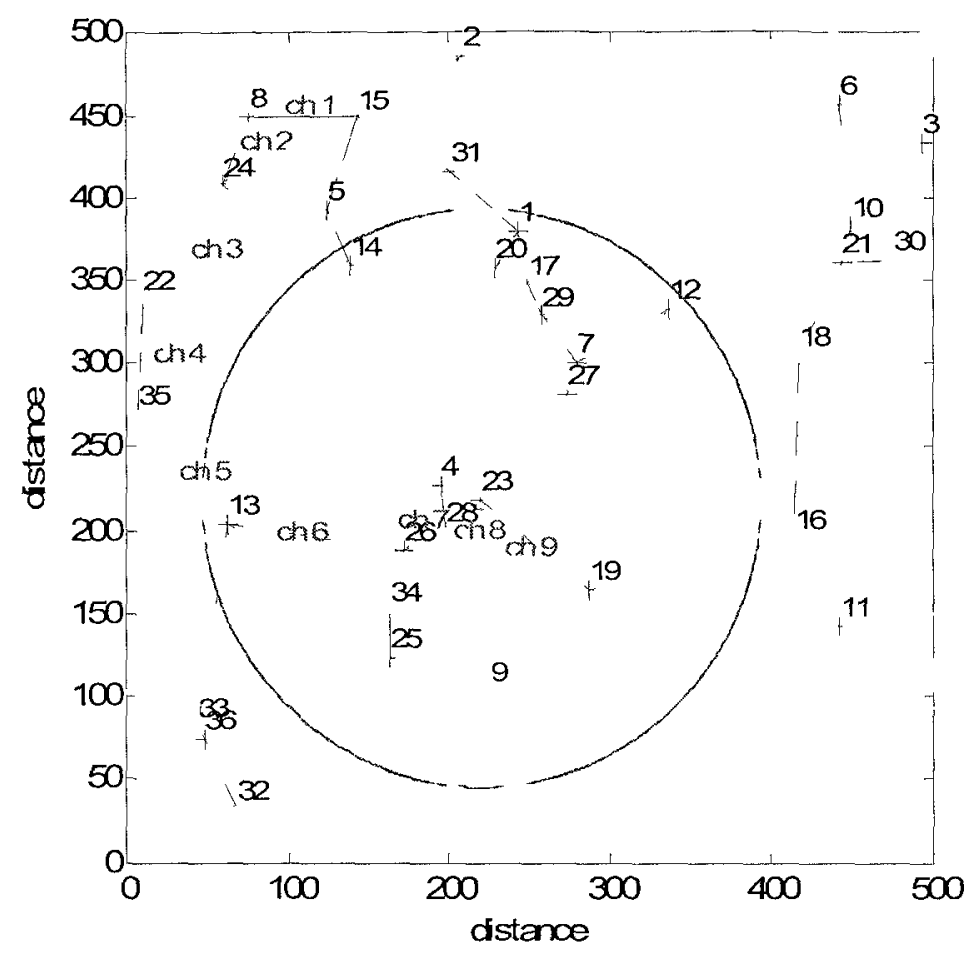

Figure 12 Interference Range of Node 23

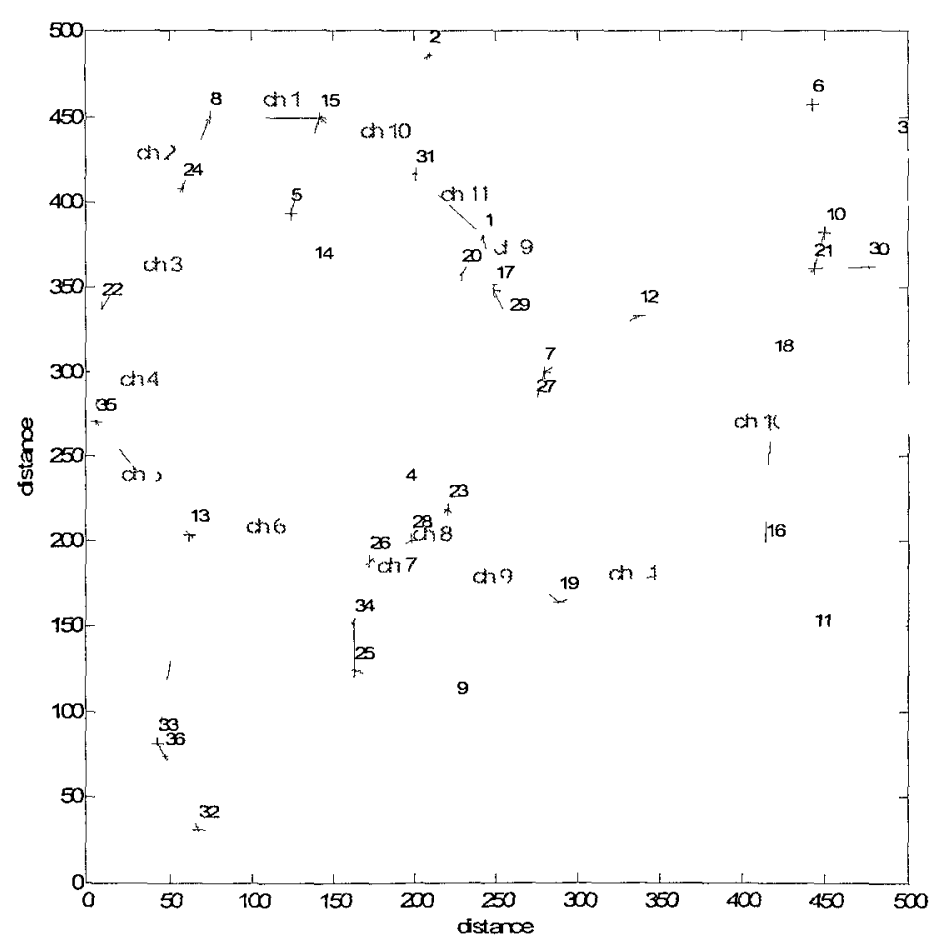

Figure 13 Interference Range of Node 17 


\section{4 e-TICA2}

Similar to e-TICA, e-TICA2 is also a centralized and fixed CAA. Its operation employs the same phases as e-TICA, however, building the minimum power based tree phase of e-TICA2 is different from e-TICA. The following sections give a detailed explanation of the modifications that have been done to e-TICA to yield a better CAA.

\subsubsection{Reduction in Interference: Minimum Spanning Tree}

Motivation: The modification done to TICA, namely 2-way interference range edge-coloring results in an enhanced CAA called e-TICA. The 2-way interference range edge-coloring leads to an improved channel assignment scheme and eliminates the problem of hidden links. This results in a better CAA. However, in some topologies, owing to the long links, LICs result in increasing the interference. Hence, reuse of a channel within the interference range causes significant decrease in network throughput.

Since the long links contribute to interference, they should be replaced with shorter links wherever possible. So, a modified CAA, e-TICA2 is presented in this section which employs a MST rooted at the gateway instead of a SPT to reduce the occurrence of conflicting channels and to improve network throughput. MST leads to more but shorter hops instead of few but longer hops in SPT. Hence, utilizing MST for topology control subsequently leads to minimal co-channel interference thereby increasing spatial channel reuse.

e-TICA2: Since transmit power is proportional to the square of the distance between the nodes, the shorter the distance, the smaller the transmit power required. This results in shrinking the interference range which implies better spatial channel reuse.

All three CAAs, TICA, e-TICA and e-TICA2 use topology control to build a connectivity graph and apply power control at mesh nodes to reduce interference and increase spatial reuse. However, unlike TICA and e-TICA which use the SPT approach, e-TICA2 uses the MST approach for building the minimum power based tree with a maximum node degree of four. During the channel assignment phase, links that are ranked higher are given priority for channel assignment. Links are ranked in decreasing order of the number of nodes that use those links to reach the gateway. If the gateway cannot find any non-conflicting channel to assign to a link, it selects an LIC. 
A scenario is investigated where a topology encounters an LIC while utilizing the e-TICA algorithm. As discussed earlier, the throughput of the network decreases due to interference caused by links using the same channel within the interference range. Random topology 17, as shown in Figure 14, has been investigated where link 18-28 has been assigned channel 5 by e-TICA and link 12-24 has also been allocated channel 5 . The circular disk in the figure indicates the interference range of node 18 . Since the link 12-24 is in the interference range of node 18 , both nodes 28 and 24 will compete for access to the medium on this channel. Specifically, when node 24 needs to communicate with node 12 on channel 5 and node 28 needs to communicate with node 18 on the same channel simultaneously, contention for medium access based on CSMA/CA will occur on channel 5. The presence of LICs affects the network throughput since some nodes, such as nodes 28 and 24 , compete for access to the medium.

A new approach is proposed for maximizing spatial channel reuse and reducing LICs by utilizing an MST rooted at the GW instead of the SPT. The motivation behind using MST is to achieve short link lengths which will result in the medium being shared efficiently by reducing LICs. Since transmit power is proportional to the distance between the nodes, the shorter the distance, the lower the transmit power. Less transmit power translates to less interference which leads to better spatial channel reuse. The modified CAA e-TICA2, replaces the SPT approach of e-TICA with the MST approach. In both approaches, the link weight is the minimum transmit power required by a node to reach its neighbor for building the minimum power based tree. It is shown in Figure 15 that utilizing MST results in shorter hops between nodes and hence, the interference range of node 18 has been shrunk. The SPT approach results in 6 LICs whereas the MST approach reduces the number of LICs in this topology to 4. Reducing LICs implies that all nodes have better access to the medium whenever they have data to transmit. Thus, competition with other nodes for access to the medium on the assigned channel is lower. So, utilizing MST will improve the network throughput and fairness in medium access. 


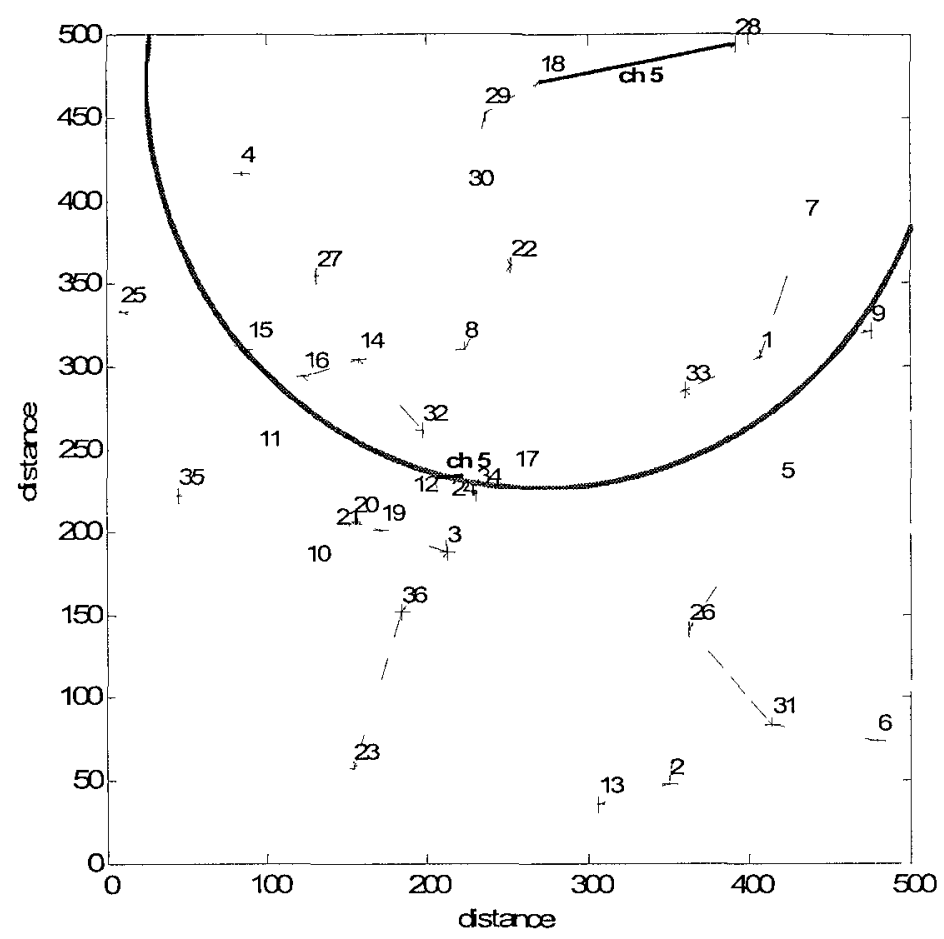

Figure 14 Interference Range of Node 18(e-TICA)

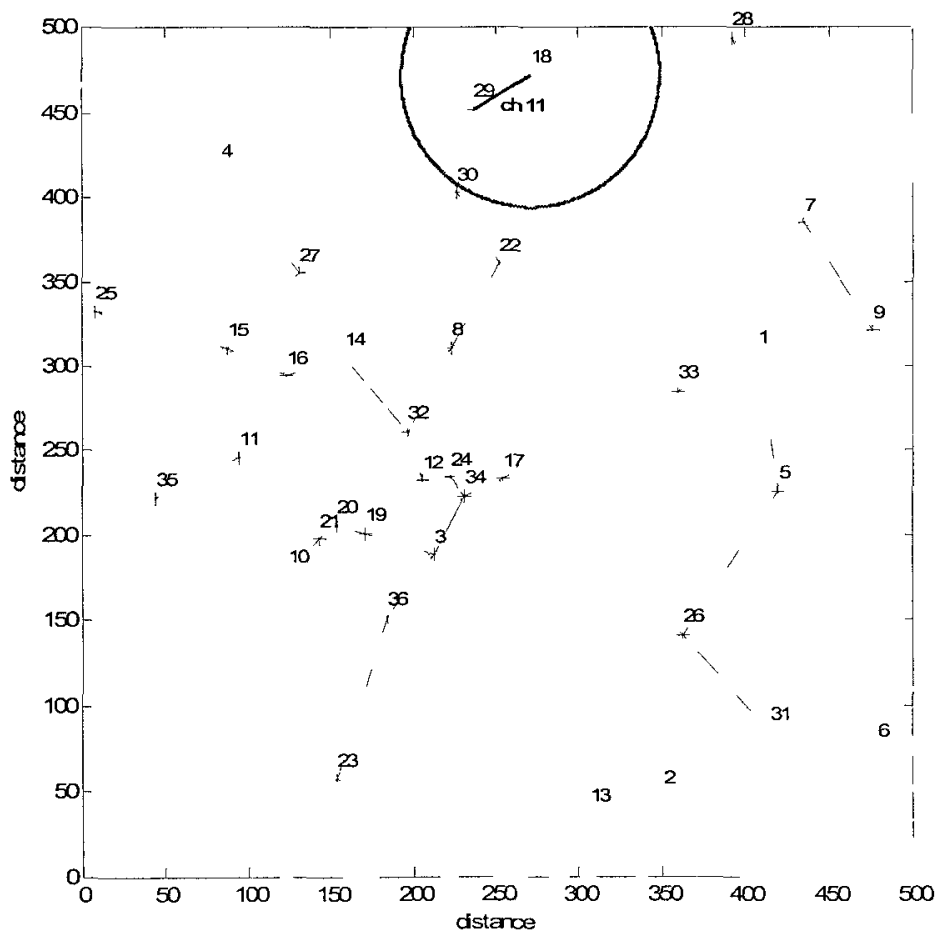

F1gure 15 Interference range of Node 18(e-TICA2) 


\subsubsection{Reduction in congestion: Utilizing the four radios of the $G W$}

Motivation: The maximum achievable throughput of a topology is limited by the performance bottleneck at the links which originate from the $\mathrm{GW}$, as well as the number of traffic sources using those links. The maximum data rates achievable at a link with one, two and three sources are 8.192 Mbps, 16.384 Mbps and 24.576 Mbps respectively. IEEE 802.11a supports a maximum data rate of $54 \mathrm{Mbps}$. However, the effective data rate is $24.748 \mathrm{Mbps}$, while the rest is consumed by overhead. Hence, if there are more than three sources sharing a link, there is a traffic bottleneck at that link with the achievable data rate being limited to 24.748 Mbps. Thus, the maximum achievable throughput can be improved by utilizing all four radios of the GW.

e-TICA2 utilizes the maximum possible radios out of the four available radios of the gateway to build a minimum spanning tree from its nearest neighbors. This approach helps in distributing the traffic load among the links that emanate from the gateway subsequently reducing congestion on those links and improving the network throughput.

e-TICA2: In e-TICA2 to fully utilize the four radios of the gateway, the algorithm has been forced to build an MST from the GW utilizing its nearest neighbors. This however, is topology dependent and utilizing all four radios might not always be possible

Random topology 14 is investigated using e-TICA and e-TICA2 as shown in Figure 16 and Figure 17 respectively. From Figure 16, it can be seen that e-TICA results in the GW utilizing only one of its four radios. This limits the maximum achievable throughput to $24.748 \mathrm{Mbps}$. As shown in Figure 16, all 13 sources are using the same link to reach the GW which causes a bottleneck at link 15-25. This traffic bottleneck limits the throughput performance of the network by confining the maximum achievable throughput. Applying e-TICA2 to the same topology increases the maximum achievable throughput to $49.3 \mathrm{Mbps}$. As shown Figure 17 e-TICA2 ensures that the GW utilizes all four of its radios. Thus, the traffic load is distributed among the four radios in e-TICA2 as compared to one radio in e-TICA. This reduces traffic congestion on the links which are close to the GW and results in an improvement in the throughput and fairness of the 
network. The throughput of random topology 14 with TICA and e-TICA is $24.6 \mathrm{Mbps}$ whereas with e-TICA2 is $49.3 \mathrm{Mbps}$.

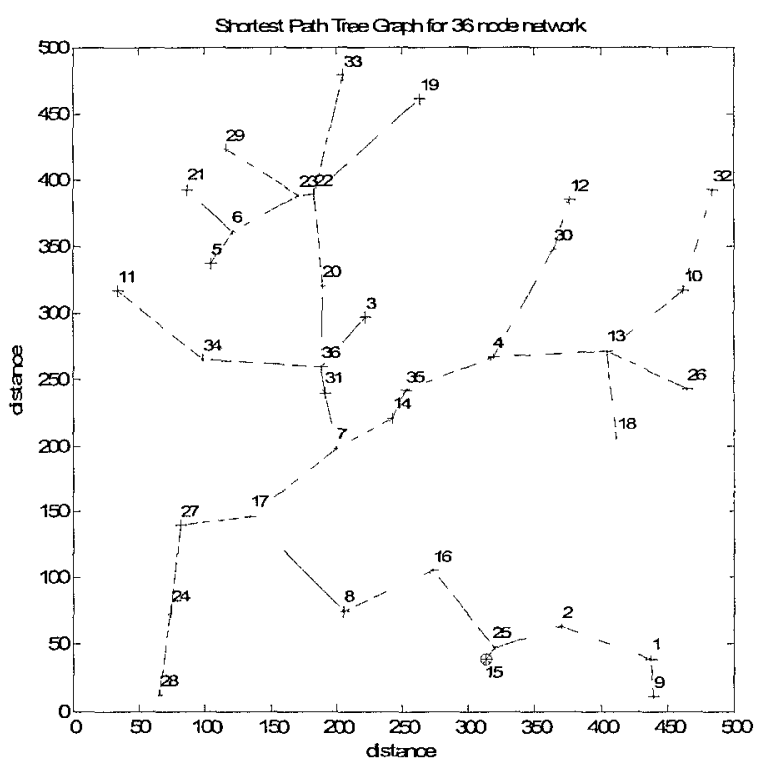

Figure 16: Shortest Path Tree (e-TICA)

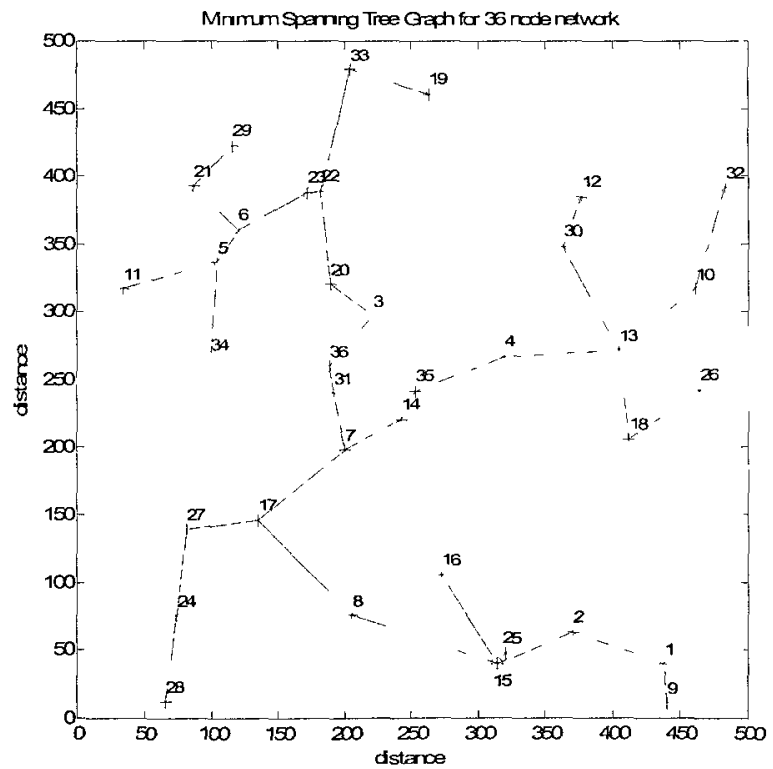

Figure 17: Minimum Spanning Tree (e-TICA2) 


\section{Chapter - 5 : Simulation Results and Evaluation}

\subsection{Introduction}

In this chapter, we describe the simulation assumptions and procedures for TICA, e-TICA and e-TICA2. We then present the simulation results for three types of topologies: (a) fixed grid, (b) controlled random and, (c) random. Then we discuss the results of the simulations and present an evaluation of all schemes relative to each other. Simulations have been done using the ns2 simulator.

\subsection{Simulation}

\subsubsection{Simulation Description}

The NS2 simulator has been used for simulation. The PHY and MAC layer settings used for the simulations in NS2 are shown in Table 5 and Table 6, respectively. The MRs at the periphery of the network are the traffic sources and send traffic to the gateway (node 15) simultaneously, thus representing a scenario in which multiple flows within the WMN interfere with each other. Each of these nodes generates an 8 Mbps Constant Bit Rate UDP traffic stream consisting of 1024 byte packets for 100 seconds.

The carrier sense threshold is twice the reception threshold which implies a carrier sensing range twice the transmission range. The interference range is equal to the carrier sensing range. The packet capture threshold is $10 \mathrm{~dB}$ and implies that if the power of the incoming packet is smaller than the power of the packet currently being received by at least the capture threshold, then the new packet is ignored. 


\subsubsection{Simulation Parameters}

Table 5: Physical Layer Parameters

\begin{tabular}{|l|l|}
\hline Physical Layer Parameters & Settings \\
\hline TX/RX Antenna Height (m) & 3 \\
\hline Gain of TX / RX Antenna & 1 \\
\hline Packet Capture Threshold (dB) & 10 \\
\hline Packet Reception Threshold (Watts) & $3.16227 \mathrm{e}-10$ \\
\hline Carrier Sense Threshold (Watts) & $7.90569 \mathrm{e}-11$ \\
\hline
\end{tabular}

Table 6: MAC layer parameters

\begin{tabular}{|l|l|}
\hline MAC Layer Parameters & Settings \\
\hline Minimum Contention Window & 15 \\
\hline Maximum Contention Window & 1023 \\
\hline Slot Time (micro seconds) & 9 \\
\hline SIFS period (micro seconds) & 16 \\
\hline Preamble Length (bits) & 96 \\
\hline PLCP Header Length (bits) & 24 \\
\hline PLCP Data Rate (Mbps) & 6 \\
\hline Basic Rate (Mbps) & 6 \\
\hline Data Rate (Mbps) & 54 \\
\hline
\end{tabular}

\subsubsection{Simulation Assumptions}

The following assumptions have been made during the simulations for all three channel assignment algorithms:

1. 36 nodes are distributed in a $500 \mathrm{~m} \times 500 \mathrm{~m}$ area.

2. Irrespective of its location, node 15 is set to be the gateway for all topologies.

3. There is a single gateway for each realization

4. Some nods are traffic generators while some are just relays. We assume that sources and relays have similar characteristics in terms of power and range.

5. All nodes have a reliable power source

6. The propagation model is chosen to be the two-ray propagation model if the distance between two nodes is greater than the cross over distance and the free space propagation model otherwise. 


\subsection{Performance Metrics for e-TICA and e-TICA2}

In this chapter, the performance of the three CAAs TICA, e-TICA and e-TICA2 has been compared based on the following criteria:

1. The total number of LICS found by each CAA. The number of LICs will have an effect on the throughput and fairness of the network since nodes sharing the same channel will contend with each other for medium access.

2. The 'Throughput Ratio', $\mathrm{T}_{\mathrm{R}}$, which is defined as the ratio of the throughput achieved by e-TICA2, e-TICA and TICA over the maximum achievable throughput in each case. $T_{R}=1$ indicates that the algorithm has achieved the maximum achievable throughput for that particular random topology.

3. The 'Fairness Ratio', $F_{R}$, which is a relative measure of the fairness achieved by the three strategies and is defined as

$$
F_{X, Y}=\frac{F_{J, X}}{F_{J, Y}}
$$

Where $\mathrm{X}$ and $\mathrm{Y}$ could be any one the three schemes

$F_{X, Y}>1$ indicates better fairness with scheme $X$ compared to scheme $Y$. The Jain's fairness index [26] is defined as:

$$
F_{J}=\frac{\left(\sum_{i=1}^{N} x_{i}\right)^{2}}{N \cdot \sum_{t=1}^{N} x_{i}^{2}}
$$

Where $x_{i}$ is the throughput of a flow $i$ and $N$ is the total number of flows (sources) in the network.

Absolute fairness is achieved when $F_{J}=1$.

\subsection{Accurate Channel Assignment: e-TICA}

The first enhancement done to TICA is the introduction of two-way interference range edge coloring. The original algorithm TICA uses one-way interference range edge coloring and does not find all the LICs in most cases. This leads to undetected hidden links which results in the CAA allocating the same channel to two links within the interference range of each others' end nodes. Using the two-way interference range edge coloring method, the modified algorithm, e-TICA, is successful in identifying all LICs. 
This leads to a better channel assignment thus reducing interference and improving fairness among flows without sacrificing the network throughput.

In this section, we will discuss the results for 25 different random and controlled random topologies.

\subsubsection{Results for Random topologies:}

\subsubsection{A Number of LICs}

Table 7 show a comparison of the number of LICs found using TICA and eTICA for twenty-five different random topologies. As can be seen from the figure, eTICA successfully finds all LICs for all 25 random topologies. For example as shown in Table 7, in random topology 2, TICA indicates that there are 5 LICs whereas, e-TICA indicates that there are $8 \mathrm{LICs}$ and accordingly allocates channels to minimize co-channel interference. Since assignment of channels is aimed at reducing the interfering environment, correctly identifying LICs is extremely crucial for proper channel assignment.

\subsubsection{B Network Throughput}

In Figure 18, the throughput ratio $\left(\mathrm{T}_{\mathrm{R}}\right)$ of e-TICA and TICA over the maximum achievable throughput for twenty-five different realizations of the random topology is shown. The difference in $T_{R}$ achieved by TICA and e-TICA is apparent from the figure. There is a higher $T_{R}$ by e-TICA in most of the random topologies. The average $T_{R}$ achieved by TICA is 0.87 whereas that achieved by e-TICA is 0.91 both with a standard deviation of 0.12 .

\subsubsection{Fairness among Flows}

In Figure 19, the fairness ratio $\left(F_{R}\right)$ among traffic flows in the network using TICA is compared with that achieved using e-TICA for twenty-five different realizations of the random topology, using

$$
F_{e-T I C A, T I C A}=\frac{F_{J, e-T I C A}}{F_{J, T I C A}}
$$


$F_{\text {e-TICATICA }}>1$ indicates better fairness by e-TICA than by TICA. It is apparent from the figure that in most of the random topologies, e-TICA again outperforms TICA in terms of fairness.

In the next two paragraphs, we will focus on two specific realizations random topology 18 and random topology 11 and show the difference in performance in these two topologies.

- Random Topology 18

Consider random topology 18 where neither TICA nor e-TICA finds any LICs. As can be observed from Figure 18 and Figure 19, the network throughput and fairness is equal for both CAAs.

- Random Topology 11

In this specific case, TICA achieves a better result in terms of throughput and fairness. 
Table 7: LICs found by TICA and e-TICA for 25 Random Topologies

\begin{tabular}{|c|c|c|}
\hline Random Topology & TICA & e-TICA \\
\hline 1 & 1 & 3 \\
\hline 2 & 5 & 8 \\
\hline 3 & 2 & 4 \\
\hline 4 & 2 & 3 \\
\hline 5 & 1 & 2 \\
\hline 6 & 2 & 2 \\
\hline 7 & 2 & 2 \\
\hline 8 & 1 & 1 \\
\hline 9 & 2 & 3 \\
\hline 10 & 2 & 3 \\
\hline 11 & 4 & 4 \\
\hline 12 & 3 & 4 \\
\hline 13 & 2 & 2 \\
\hline 14 & 3 & 3 \\
\hline 15 & 2 & 2 \\
\hline 16 & 3 & 3 \\
\hline 17 & 4 & 6 \\
\hline 18 & 0 & 0 \\
\hline 19 & 1 & 1 \\
\hline 20 & 2 & 3 \\
\hline 21 & 1 & 3 \\
\hline 22 & 2 & 3 \\
\hline 23 & 2 & 2 \\
\hline 24 & 4 & 4 \\
\hline 25 & 3 & 4 \\
\hline
\end{tabular}

Table 8: Results for number of LICs for 25 Random Topologies

\begin{tabular}{|c|c|c|}
\hline CAA Topology & $\begin{array}{c}\text { Average number of } \\
\text { LICs }\end{array}$ & $\begin{array}{c}\text { 95 \% Confidence Interval } \\
\text { for number of LICs }\end{array}$ \\
\hline TICA & 2.24 & $1.79-2.69$ \\
\hline e-TICA & 3.00 & $2.38-3.62$ \\
\hline
\end{tabular}




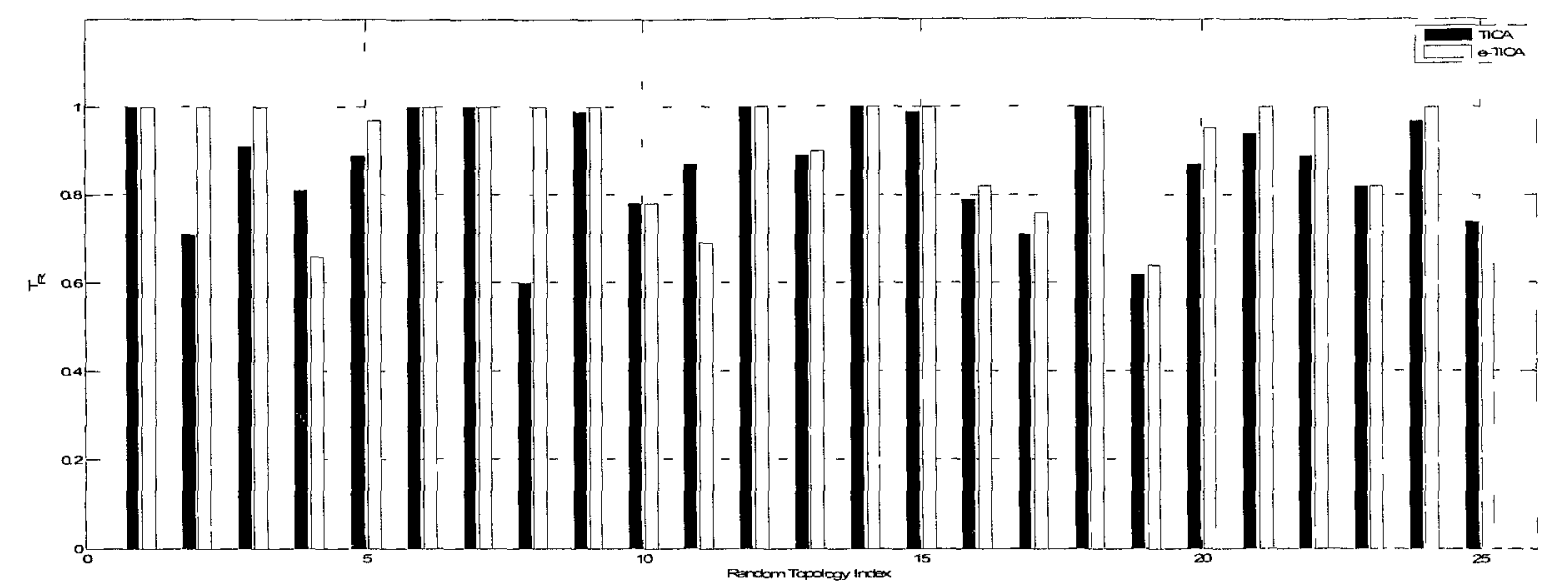

Figure 18: Comparison of Throughput Ratio for 25 Random Topologies

Table 9: Results for Throughput Ratio for 25 Random Topologies

\begin{tabular}{|c|c|c|}
\hline CAA & $\begin{array}{c}\text { Average Throughput } \\
\text { Ratio }\end{array}$ & $\begin{array}{c}\mathbf{9 5} \% \text { Confidence Interval } \\
\text { for Throughput Ratio }\end{array}$ \\
\hline TICA & 0.87 & $0.82-0.92$ \\
\hline e-TICA & 0.91 & $0.86-0.96$ \\
\hline
\end{tabular}

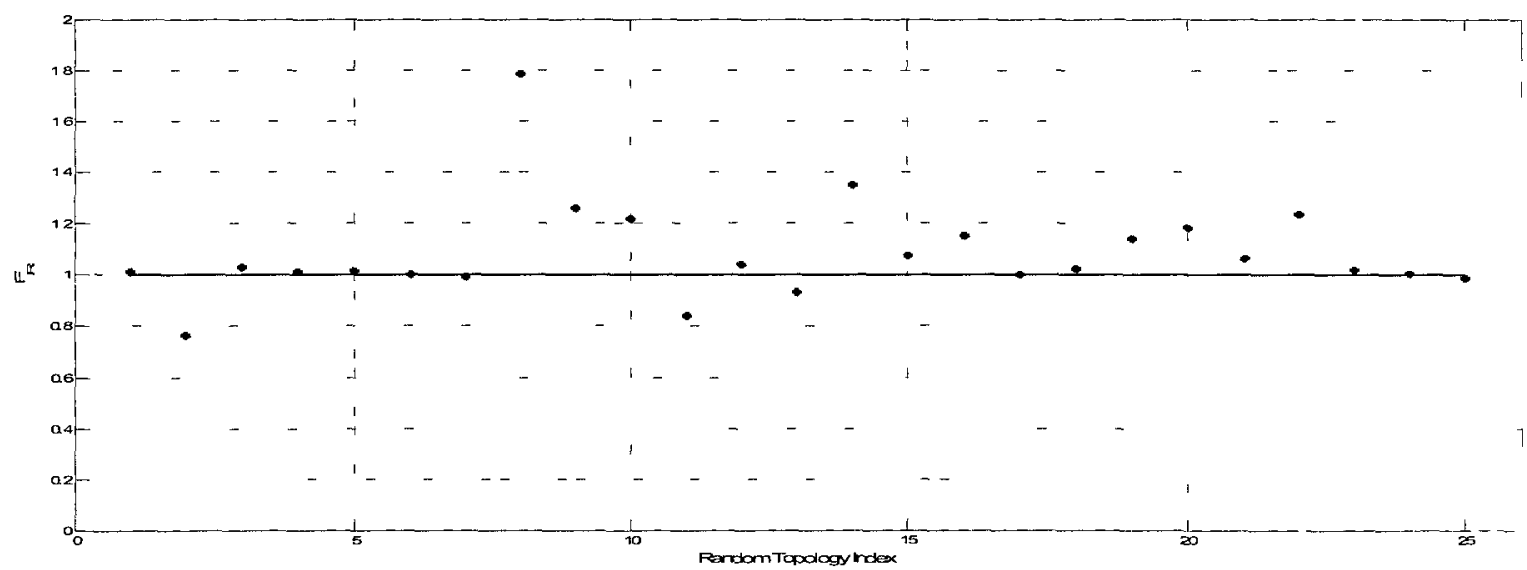

Figure 19: Comparison of Fairness Ratio for 25 Random Topologies

Table 10: Results for Fairness Ratio of 25 Random Topologies

\begin{tabular}{|c|c|c|}
\hline CAA & $\begin{array}{c}\text { Average Fairness } \\
\text { Ratio }\end{array}$ & $\begin{array}{l}\text { 95\% Confidence Interval } \\
\text { for Fairness Ratio }\end{array}$ \\
\hline $\begin{array}{l}\text { e-TICA over } \\
\text { TICA }\end{array}$ & 1.08 & $1.01-1.16$ \\
\hline
\end{tabular}




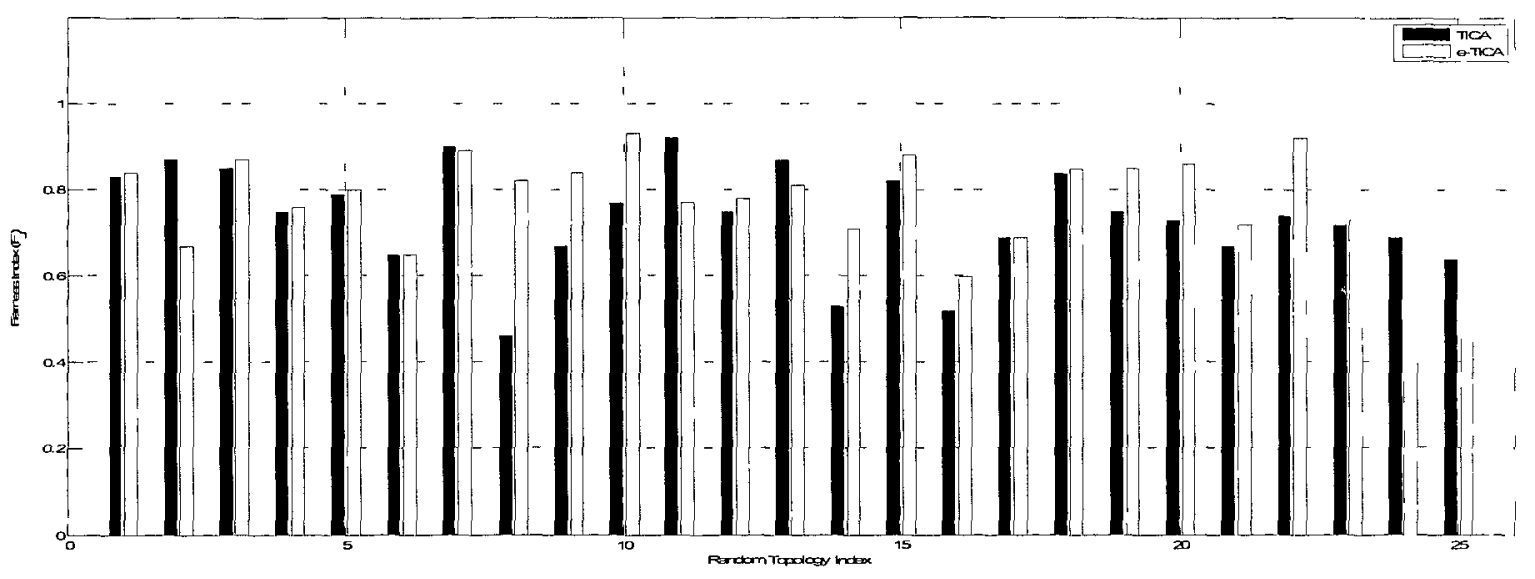

Figure 20: Jain's Fairness Index for 25 Random Topologies

Table 11: Results for Jain's Fairness Index for Random Topologies

\begin{tabular}{|c|c|c|}
\hline TAA & $\begin{array}{c}\text { Average Fairness } \\
\text { Index }\end{array}$ & $\begin{array}{l}\text { 95\% Confidence Interval } \\
\text { for Fairness Index }\end{array}$ \\
\hline TICA & 0.74 & $0.69-0.78$ \\
\hline e-TICA & 0.78 & $0.75-0.82$ \\
\hline
\end{tabular}




\subsubsection{Results for Controlled Random topologies:}

\subsubsection{A Number of LICS}

Table 12 shows a comparison of the number of LICs found using TICA and eTICA for twenty-five different controlled random topologies. As can be seen from the table, e-TICA successfully finds all LICs for all 25 controlled random topologies. For example as shown in Table 12, in controlled random topology 5, TICA indicates that there are 5 LICs whereas, e-TICA indicates 7 LICs and accordingly allocates channels to links to minimize co-channel interference. Since assignment of channels is aimed at reducing the interfering environment, correctly identifying LICs is extremely crucial for proper channel assignment.

\subsubsection{B Network Throughput}

In Figure 21, the throughput ratio $\left(T_{R}\right)$ of e-TICA and TICA over the maximum achievable throughput for twenty-five different realizations of the controlled random topology is shown. The difference in $T_{R}$ achieved by TICA and e-TICA is apparent from the figure. There is higher $T_{R}$ by e-TICA in most of the controlled random topologies. The average $T_{R}$ achieved by TICA is 0.88 whereas that achieved by e-TICA is 0.93 both with a standard deviation of 0.08 .

\subsubsection{Fairness among Flows}

In Figure 22, the fairness ratio $\left(\mathrm{F}_{\mathrm{R}}\right)$ among traffic flows in the network using TICA is compared with that achieved using e-TICA for twenty-five different realizations of the controlled random topology, using (3).

As discussed in section 5.3, $F_{R}>1$ indicates better fairness by e-TICA than by TICA. It is apparent from Figure 22 that in most of the random topologies, e-TICA again outperforms TICA in terms of fairness. The average $F_{R}$ over the twenty-five random topologies is 1.03 with a standard deviation of 0.11 . 
Table 12: LICs found by TICA and e-TICA for 25 Controlled Random Topologies

\begin{tabular}{|c|c|c|}
\hline Random Topology & TICA & e-TICA \\
\hline 1 & 3 & -1 \\
\hline 2 & 2 & 3 \\
\hline 3 & 5 & 6 \\
\hline 4 & 3 & 3 \\
\hline 5 & 5 & 7 \\
\hline 6 & 4 & 4 \\
\hline 7 & 2 & 2 \\
\hline 8 & 3 & 3 \\
\hline 9 & 2 & 1 \\
\hline 10 & 6 & 7 \\
\hline 11 & 5 & 4 \\
\hline 12 & 1 & 1 \\
\hline 13 & 5 & 5 \\
\hline 14 & 4 & 4 \\
\hline 15 & 2 & 5 \\
\hline 16 & 1 & 2 \\
\hline 17 & 2 & 3 \\
\hline 18 & 3 & 2 \\
\hline 19 & 5 & 5 \\
\hline 20 & 6 & 7 \\
\hline 21 & 3 & 4 \\
\hline 22 & 7 & 8 \\
\hline 23 & 6 & 7 \\
\hline 24 & 2 & 3 \\
\hline 25 & 2 & 4 \\
\hline
\end{tabular}

Table 13: Results for number of LICs for Controlled Random Topologies

\begin{tabular}{|c|c|c|}
\hline CAA & $\begin{array}{c}\text { Average number of } \\
\text { LICs }\end{array}$ & $\begin{array}{c}95 \% \text { Confidence Interval } \\
\text { for number of LICs }\end{array}$ \\
\hline TICA & 3.56 & $2.89-4.23$ \\
\hline e-TICA & 4.16 & $3.40-4.90$ \\
\hline
\end{tabular}




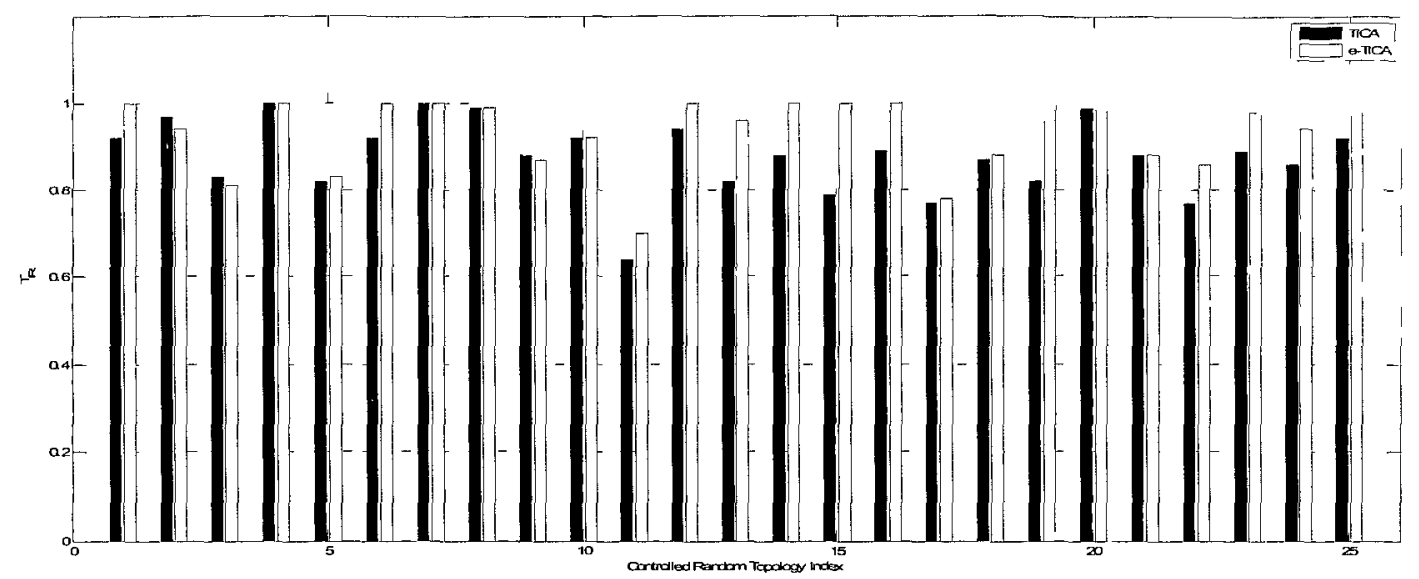

Figure 21: Comparison of Throughput Ratio for 25 Controlled Random Topologies

Table 14: Results for Throughput Ratio of Controlled Random Topologies

\begin{tabular}{|c|c|c|}
\hline CAA & $\begin{array}{c}\text { Average Throughput } \\
\text { Ratio }\end{array}$ & $\begin{array}{c}\text { 95\% Confidence Interval } \\
\text { for Throughput Ratio }\end{array}$ \\
\hline TICA & 0.88 & $085-0.91$ \\
\hline e-TICA & 0.93 & $090-0.97$ \\
\hline
\end{tabular}

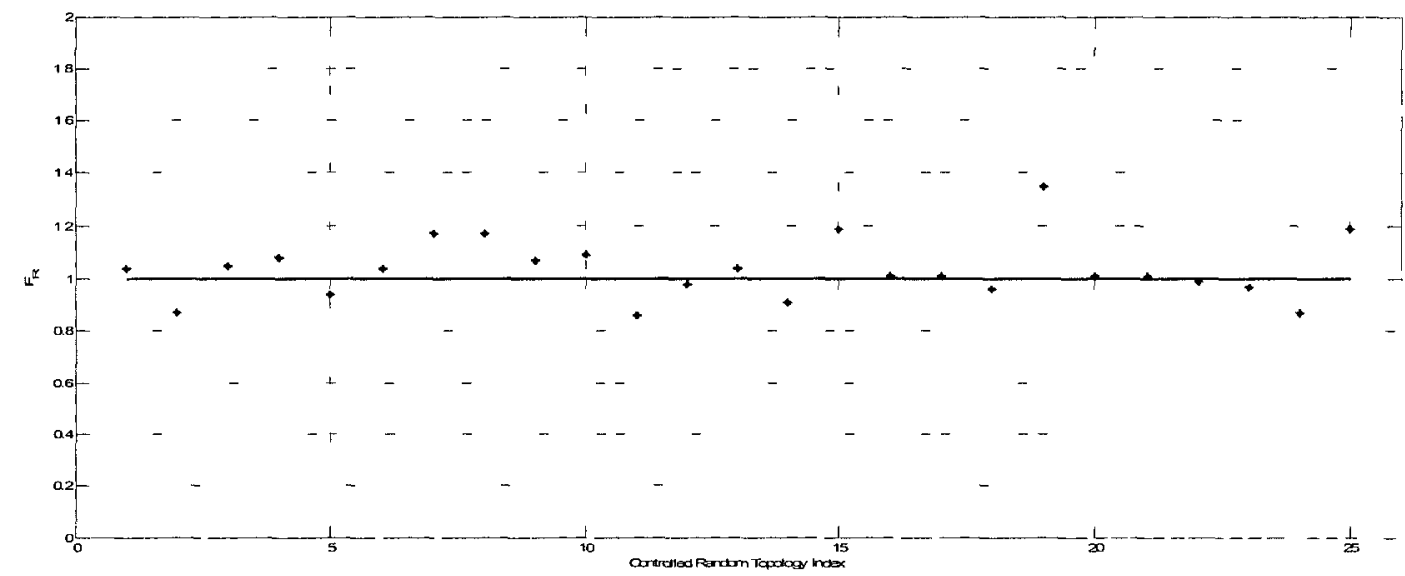

Figure 22: Comparison of Fairness Ratio for 25 Controlled Random Topologies

Table 15: Results for Fairness ratio of Controlled Random Topologies

\begin{tabular}{|l|c|c|}
\hline CAA & $\begin{array}{c}\text { Average Fairness } \\
\text { Ratio }\end{array}$ & $\begin{array}{r}95 \% \text { Confidence } \\
\text { Interval for Fairness Ratio }\end{array}$ \\
\hline e-TICA over TICA & 1.03 & $099-1.08$ \\
\hline
\end{tabular}




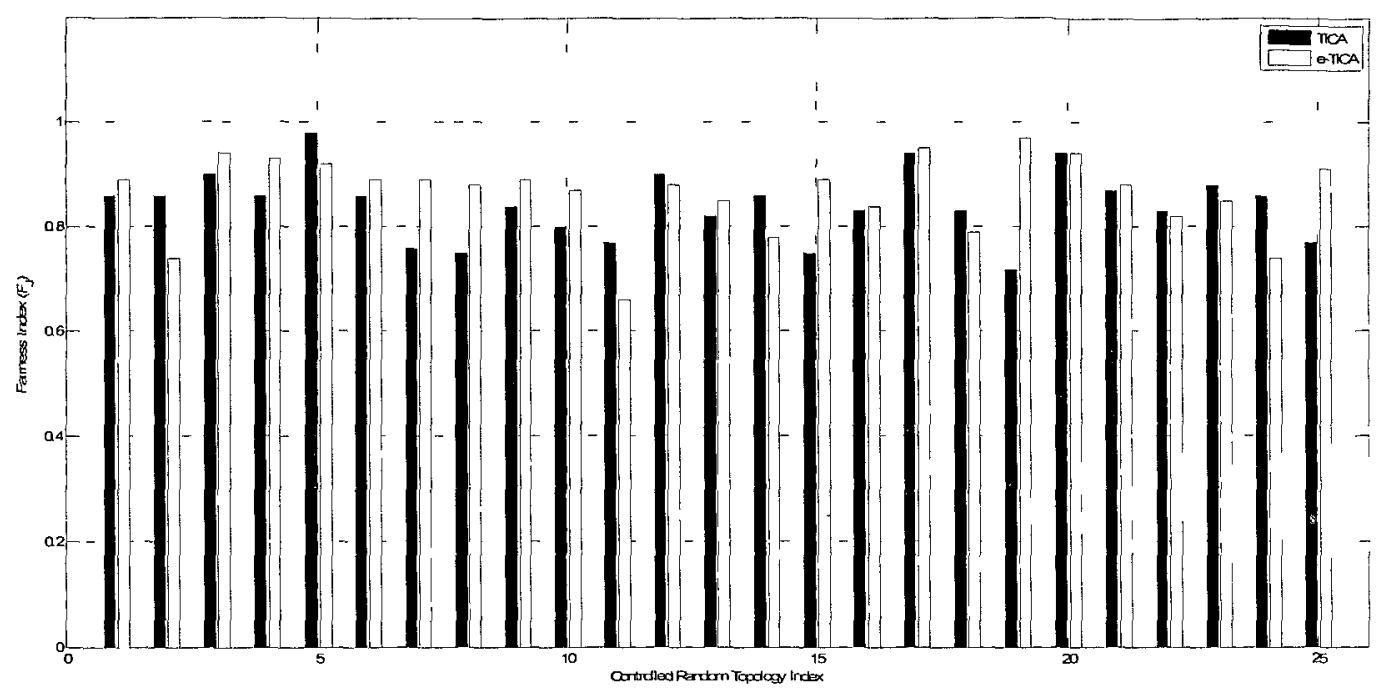

Figure 23: Jain's Fairness Index for 25 Controlled Random Topologies Table 16: Results for Jain`s Fairness Index

\begin{tabular}{|c|c|c|}
\hline Topology & $\begin{array}{c}\text { Average Jain`s } \\
\text { Fairness Index }\end{array}$ & $\begin{array}{c}\mathbf{9 5} \% \text { Confidence Interval } \\
\text { for Jain`s Fairness Index }\end{array}$ \\
\hline TICA & 0.84 & $0.82-0.86$ \\
\hline e-TICA & 0.86 & $0.84-0.89$ \\
\hline
\end{tabular}




\subsection{Reducing interference and congestion: eTICA2}

The presence of long links in some topologies leads to increased co-channel interference. This causes a decrease in throughput and fairness since some nodes which operate on a conflicting channel contend with each other for channel access. A minimum spanning tree rooted at the gateway replaces the long links with shorter ones. Shorter hops lead to shrinking the interference range which in turn leads to better spatial reuse.

TICA and e-TICA use the shortest path tree approach to build a minimum power based tree from the gateway to each node whereas e-TICA2 employs the minimum spanning tree approach for the same.

The traffic bottleneck at the GW limits the throughput performance of the network by confining the maximum achievable throughput of the network. e-TICA2 ensures that the GW utilizes the maximum possible radios out of its four available radios to build an MST. The traffic load is now distributed among the links of the GW. The number of radios that the GW can utilize depends on the number of its nearest neighbors.

In this section, for a fair comparison, we have ensured that the number of traffic sources is the same for all three CAAs in the following way:

If $\mathrm{A}=$ \{end nodes for SPT $\}$ and $\mathrm{B}=$ \{end nodes of MST $\}$, then for comparing all three CAAs, we have made a super set ' $\mathrm{C}$ ' which is defined as

$$
\mathrm{C}=\mathrm{A} \cup \mathrm{B} \text {. }
$$

Hence, $\mathrm{C}=$ \{end nodes of SPT and MST $\}$

Thus, the traffic sources in each realization of the random topology for each CAA, are the end nodes of the SPT and the end nodes of the MST.

The results in the following section show that e-TICA2 outperforms both TICA and e-TICA in terms of network throughput without compromising the fairness.

\subsubsection{Random Topologies}

\subsubsection{A Number of LICs}

Table 17 shows the number of LICs encountered by TICA, e-TICA and e-TICA2 for 25 random topologies. Table 17 indicates that among the three CAAs, e-TICA2 
encounters the lowest number of LICs in most random topologies. This affects the spatial reuse of channels and therefore the network throughput.

\subsubsection{B Network Throughput}

Figure 25, which shows a comparison of the throughput ratio $\left(T_{R}\right)$ for the three CAAs, indicates that e-TICA2 not only outperforms TICA and e-TICA in most random topologies but achieves the maximum achievable throughput for many realizations.

\subsubsection{Fairness among Flows}

Figure 26 shows that fairness ratio of e-TICA2 is comparable to e-TICA and better than TICA. The average fairness ratio of e-TICA2 over e-TICA is 0.98 with a standard deviation of 0.19 and the average fairness ratio of e-TICA2 over TICA is 1.05 with a standard deviation of 0.2 .

Sometimes, the location of the gateway will produce unexpected results. Consider for example, random topology 19. Table 17 indicates that this topology does not encounter any LICs with e-TICA2. Hence, there is fairness in medium access for this topology. From Figure 25 , we can see that the throughput ratio for e-TICA2 is 0.99 which means that e-TICA2 achieves the maximum achievable throughput for this topology. However, from Figure 27 we can see that the fairness index of this topology is 0.75. This happens because the topology built is such that there are two links emanating from the GW and 7 sources share the same link. Thus there is congestion at the link 15 19 and hence, per flow fairness suffers.

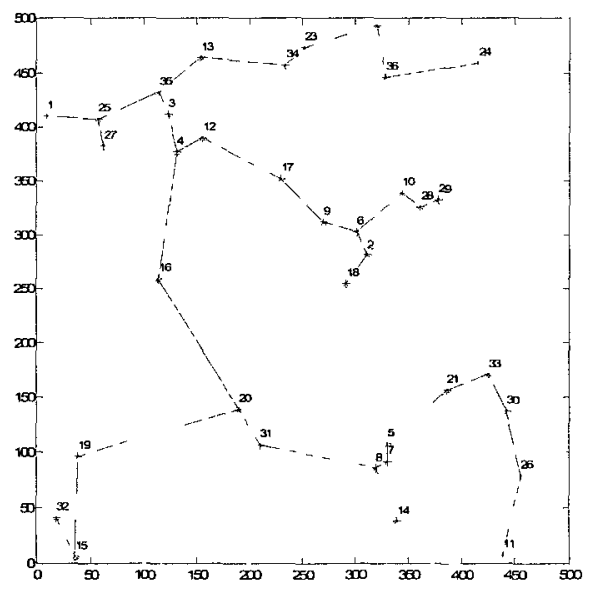

Figure 24: Random topology 19 eTICA-2 
Table 17: LICs found in 25 Random Topologies by TICA, e-TICA and e-TICA2

\begin{tabular}{|c|c|c|c|}
\hline Random Topology & TICA & e-TICA & e-TICA2 \\
\hline 1 & 1 & 3 & 1 \\
\hline 2 & 5 & 8 & 3 \\
\hline 3 & 2 & 4 & 1 \\
\hline 4 & 2 & 3 & 3 \\
\hline 5 & 1 & 2 & 2 \\
\hline 6 & 2 & 2 & 2 \\
\hline 7 & 2 & 2 & 2 \\
\hline 8 & 1 & 1 & 2 \\
\hline 9 & 2 & 3 & 1 \\
\hline 10 & 2 & 3 & 1 \\
\hline 11 & 4 & 4 & 3 \\
\hline 12 & 3 & 4 & 2 \\
\hline 13 & 2 & 2 & 1 \\
\hline 14 & 3 & 3 & 1 \\
\hline 15 & 2 & 2 & 2 \\
\hline 16 & 3 & 3 & 2 \\
\hline 17 & 4 & 6 & 4 \\
\hline 18 & 0 & 0 & 1 \\
\hline 19 & 1 & 1 & 0 \\
\hline 20 & 2 & 3 & 1 \\
\hline 21 & 1 & 3 & 1 \\
\hline 22 & 2 & 3 & 2 \\
\hline 23 & 2 & 2 & 1 \\
\hline 24 & 4 & 4 & 1 \\
\hline 25 & 3 & 4 & 3 \\
\hline
\end{tabular}

Table 18: Results for number of LICs for Random Topologies

\begin{tabular}{|l|c|c|}
\hline CAA & $\begin{array}{c}\text { Average number of } \\
\text { LICs }\end{array}$ & $\begin{array}{c}\text { 95 \% Confidence } \\
\text { Interval for number of LIcs }\end{array}$ \\
\hline TICA & 2.24 & $1.79-2.69$ \\
\hline e-TICA & 3.00 & $2.38-3.62$ \\
\hline e-TICA2 & 1.72 & $1.36-2.08$ \\
\hline
\end{tabular}




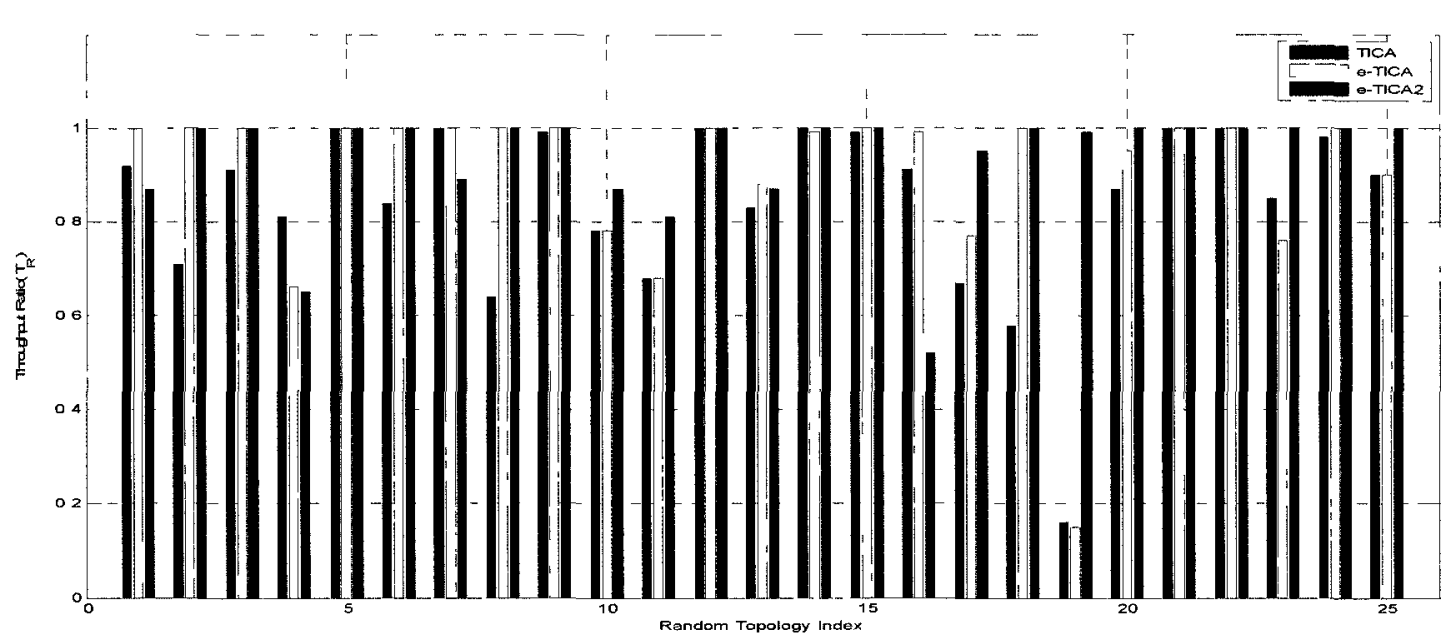

Figure 25: Comparison of Throughput Ratio of 25 Random Topologies

Table 19: Results for Throughput Ratio for Random Topologies

\begin{tabular}{|l|c|c|}
\hline CAA & $\begin{array}{c}\text { Average Throughput } \\
\text { Ratio }\end{array}$ & $\begin{array}{c}\text { 95 \% Confidence Interval } \\
\text { for Throughput ratio }\end{array}$ \\
\hline TICA & 0.84 & $0.77-0.91$ \\
\hline e-TICA & 0.90 & $0.83-0.97$ \\
\hline e-TICA2 & 0.94 & $0.89-0.98$ \\
\hline
\end{tabular}

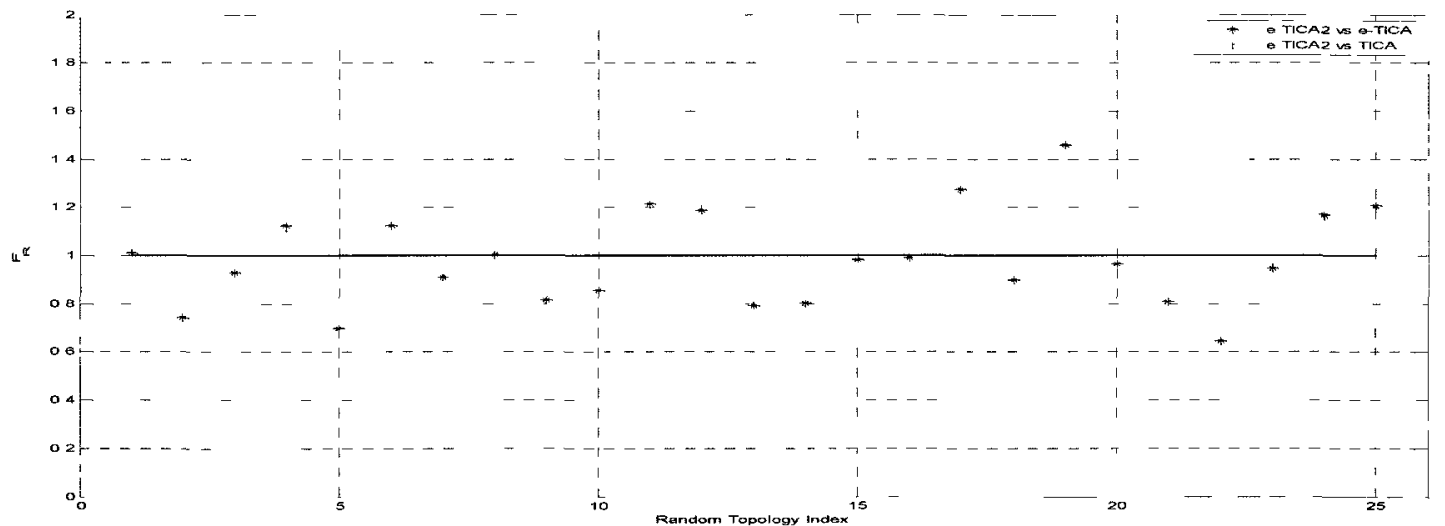

Figure 26: Comparison of Fairness ratio of 25 Random Topologies

Table 20: Results for Fairness Ratio for Random Topologies

\begin{tabular}{|l|c|c|}
\hline CAA & $\begin{array}{c}\text { Average Fairness } \\
\text { Ratio }\end{array}$ & $\begin{array}{c}\text { 95\% Confidence } \\
\text { Interval for Fairness Ratio }\end{array}$ \\
\hline e-TICA2 over e-TICA & 0.98 & $0.91-1.06$ \\
\hline e-TICA2 over TICA & 1.05 & $0.97-1.13$ \\
\hline
\end{tabular}




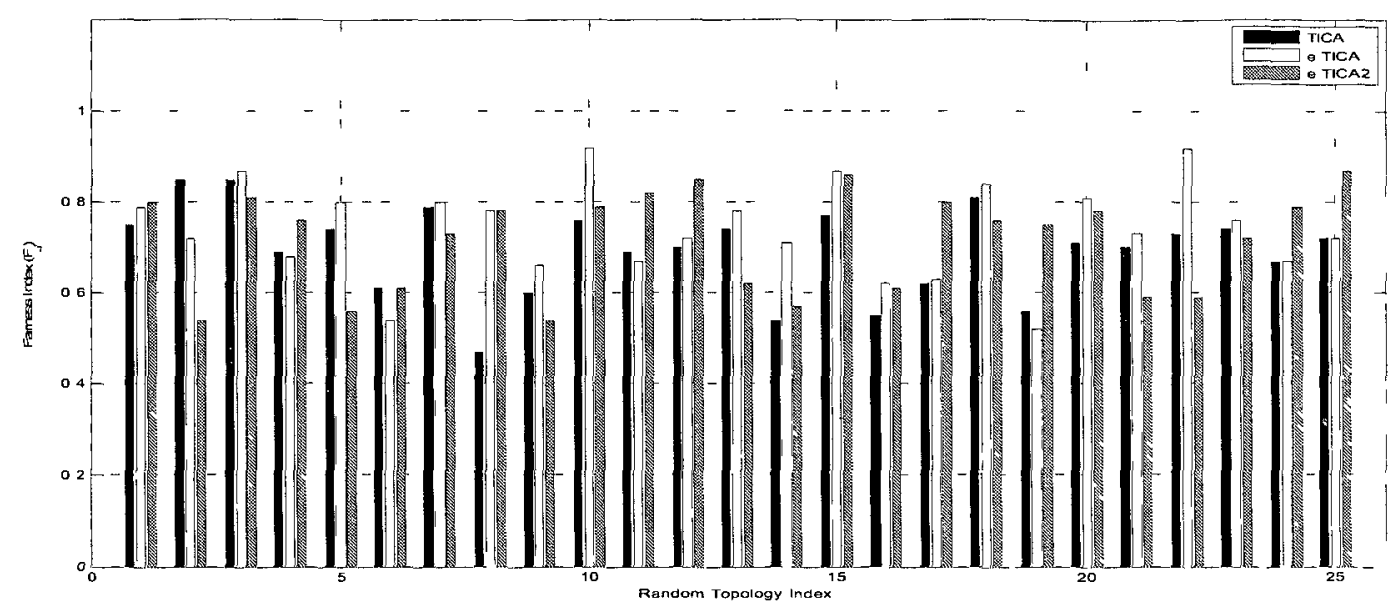

Figure 27: Fairness Index for all 3 CAAs for Random Topologies

Table 21: Results for Jain`s Fairness Index for Random Topologies

\begin{tabular}{|c|c|c|}
\hline & $\begin{array}{c}\text { Average Jain`s Fairness } \\
\text { Index }\end{array}$ & $\begin{array}{c}95 \% \text { Confidence Interval } \\
\text { for Jain`s Fairness Index }\end{array}$ \\
\hline TICA & 0.69 & $0.66-0.73$ \\
\hline e-TICA & 0.74 & $0.70-0.78$ \\
\hline e-TICA2 & 0.72 & $0.67-0.76$ \\
\hline
\end{tabular}




\subsubsection{Controlled Random Topologies}

\subsubsection{A Number of LICs}

Table 22 shows the number of LICs encountered by TICA, e-TICA and e-TICA2 for 25 controlled random topologies. The table indicates that among the three CAAs, eTICA2 encounters the lowest number of LICs in most random topologies. This affects the spatial reuse of channels and therefore the network throughput.

\subsubsection{B Network Throughput}

Figure 28, which shows a comparison of the throughput ratio $\left(T_{R}\right)$ for the three CAAs, indicates that e-TICA2 is comparable to e-TICA and better than TICA in most controlled random topologies.

\subsubsection{Fairness among Flows}

Figure 29 shows that fairness ratio of e-TICA2 is comparable to e-TICA and better than TICA. The average fairness ratio of e-TICA2 over e-TICA is 0.98 with a standard deviation of 0.19 and the average faimess ratio of e-TICA2 over TICA is 1.05 with a standard deviation of 0.2 . 
Table 22: LICs found in 25 Controlled Random Topologies by TICA, e-TICA and eTICA2

\begin{tabular}{|c|c|c|c|}
\hline $\begin{array}{c}\text { Controlled } \\
\text { Random Topology }\end{array}$ & TICA & e-TICA & e-TICA2 \\
\hline 1 & 3 & 4 & 2 \\
\hline 2 & 2 & 3 & 1 \\
\hline 3 & 5 & 6 & 0 \\
\hline 4 & 3 & 3 & 2 \\
\hline 5 & 5 & 7 & 4 \\
\hline 6 & 4 & 4 & 2 \\
\hline 7 & 2 & 2 & 0 \\
\hline 8 & 3 & 3 & 1 \\
\hline 9 & 2 & 1 & 1 \\
\hline 10 & 6 & 7 & 1 \\
\hline 11 & 5 & 4 & 1 \\
\hline 12 & 1 & 1 & 1 \\
\hline 13 & 5 & 5 & 1 \\
\hline 14 & 4 & 4 & 0 \\
\hline 15 & 2 & 5 & 1 \\
\hline 16 & 1 & 2 & 1 \\
\hline 17 & 2 & 3 & 0 \\
\hline 18 & 3 & 2 & 0 \\
\hline 19 & 5 & 5 & 0 \\
\hline 20 & 6 & 7 & 4 \\
\hline 21 & 3 & 4 & 2 \\
\hline 22 & 7 & 8 & 1 \\
\hline 23 & 6 & 7 & 2 \\
\hline 24 & 2 & 3 & 1 \\
\hline 25 & 2 & 4 & 2 \\
\hline
\end{tabular}

Table 23: Results for number of LICs for Controlled Random Topologies

\begin{tabular}{|l|c|c|}
\hline CAA & $\begin{array}{c}\text { Average number of } \\
\text { LICs }\end{array}$ & $\begin{array}{c}\text { 95 \% Confidence } \\
\text { Interval for number of LIcs }\end{array}$ \\
\hline TICA & 3.56 & $2.89-4.23$ \\
\hline e-TICA & 4.16 & $3.40-4.92$ \\
\hline e-TICA2 & 1.24 & $0.82-1.66$ \\
\hline
\end{tabular}




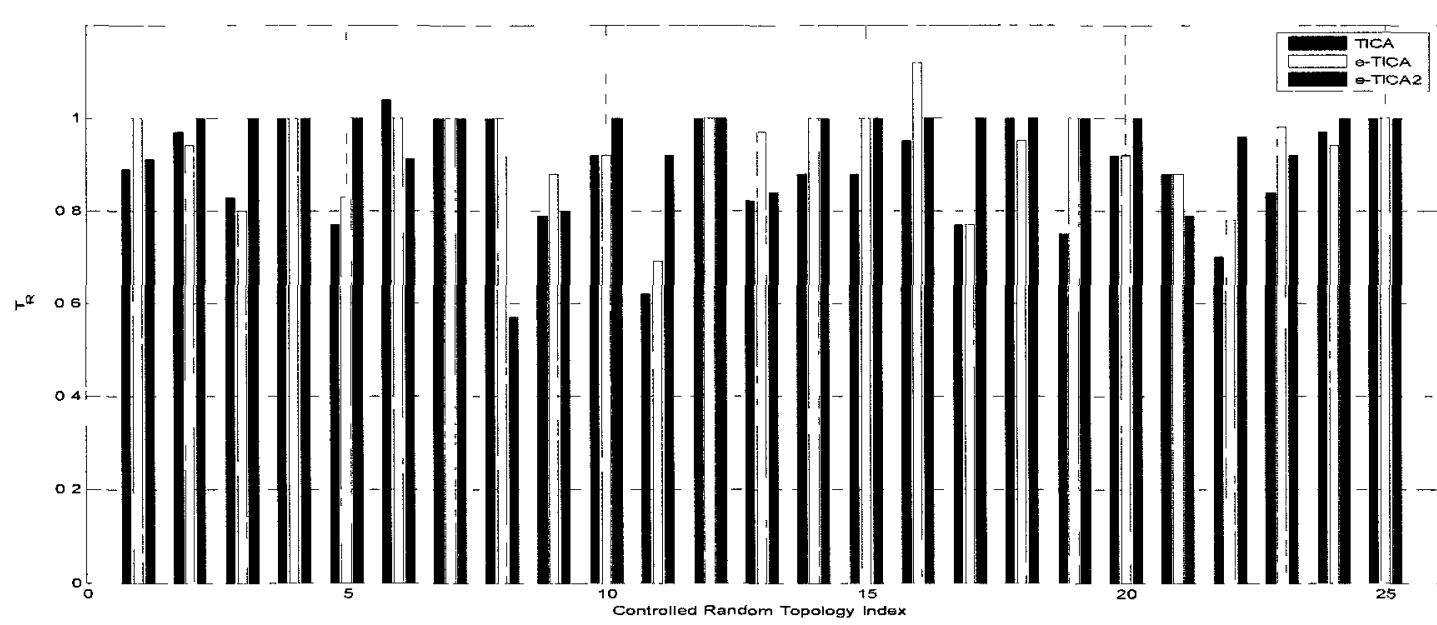

Figure 28: Comparison of Throughput Ratio of 25 Controlled Random Topologies

Table 24: Results for Throughput Ratio for Controlled Random Topologies

\begin{tabular}{|l|c|c|}
\hline CAA & $\begin{array}{c}\text { Average Throughput } \\
\text { Ratio }\end{array}$ & $\begin{array}{c}\mathbf{9 5} \% \text { Confidence Interval } \\
\text { for Throughput ratio }\end{array}$ \\
\hline TICA & 0.89 & $0.84-0.93$ \\
\hline e-TICA & 0.93 & $0.90-0.97$ \\
\hline e-TICA2 & 0.94 & $0.90-0.98$ \\
\hline
\end{tabular}

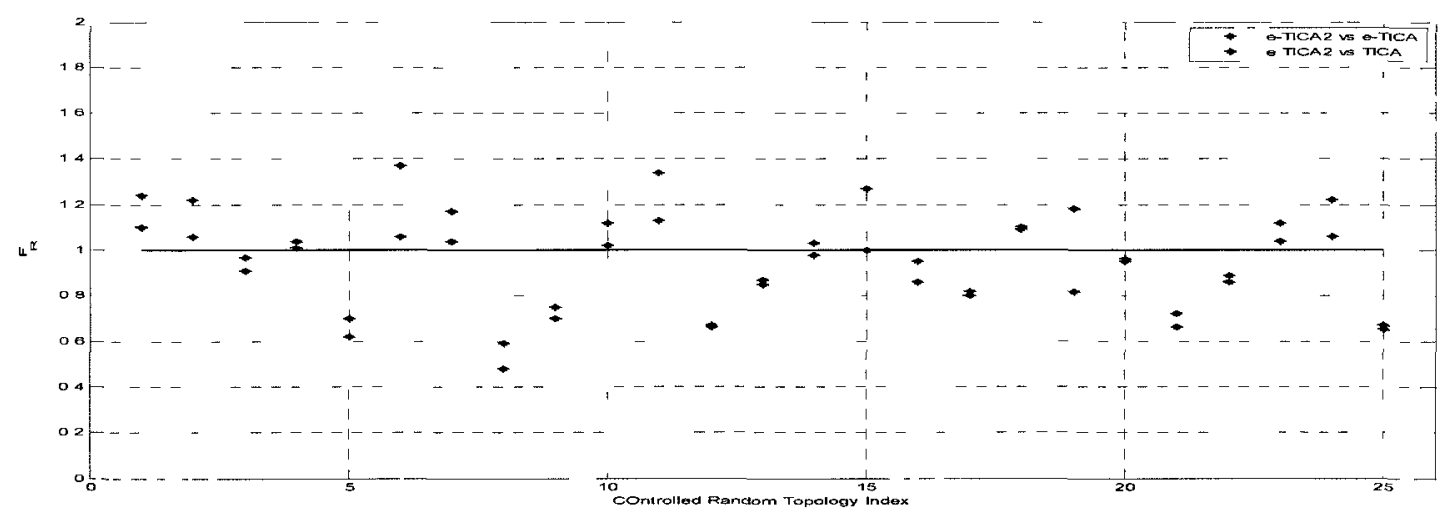

Figure 29: Comparison of Fairness ratio of 25 Controlled Random Topologies

Table 25: Results for Fairness Ratio for Controlled Random Topologies

\begin{tabular}{|l|c|c|}
\hline CAA & $\begin{array}{c}\text { Average Fairness } \\
\text { Ratio }\end{array}$ & $\begin{array}{c}\text { 95 \% Confidence } \\
\text { Interval for Fairness Ratio }\end{array}$ \\
\hline e-TICA2 over e-TICA & 0.93 & $0.84-1.01$ \\
\hline e-TICA2 over TICA & 0.97 & $0.89-1.05$ \\
\hline
\end{tabular}




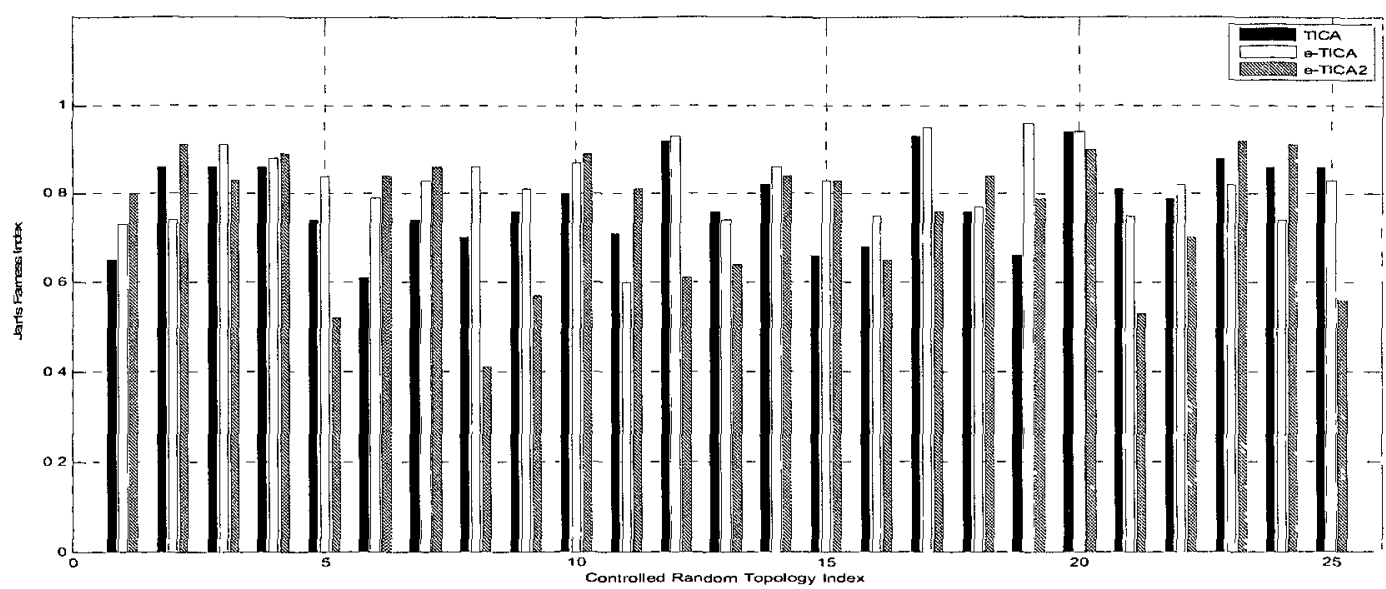

Figure 30: Fairness Index for all 3 CAAs for Controlled Random topologies

Table 26: Results for Jain's Fairness Index for Controlled Random Topologies

\begin{tabular}{|c|c|c|}
\hline & $\begin{array}{c}\text { Average Jain`s Fairness } \\
\text { Index }\end{array}$ & $\begin{array}{c}\text { 95\% Confidence Interval } \\
\text { for Jain`s Fairness Index }\end{array}$ \\
\hline TICA & 0.78 & $0.75-0.82$ \\
\hline e-TICA & 0.82 & $0.79-0.85$ \\
\hline e-TICA2 & 0.75 & $0.70-0.81$ \\
\hline
\end{tabular}




\subsection{Grid Topology}

In this section, an analysis of the results of the grid topology for all three CAAs namely TICA, e-TICA and e-TICA2, is presented. Table 11 shows the number of LICs found by the three CAAs for a grid topology of 36 nodes in an area of $500 \mathrm{mx} 500 \mathrm{~m}$. Table 10 shows the throughput ratio for the grid topology for all three CAAs.

\subsubsection{Performance evaluation of TICA}

As shown in Table 27 TICA does not encounter any LICs for the Grid topology. As shown in Table 28 the throughput ratio of the Grid topology using TICA is 1.00 which implies that the throughput achieved by TICA for the grid topology is equal to the maximum achievable throughput for that topology. The fairness index of the Grid topology using TICA is 0.88 .

\subsubsection{Performance evaluation of e-TICA}

As shown in Table 27 e-TICA also does not encounter any LICs for the Grid topology. As shown in Table 28 the throughput ratio of the Grid topology using e-TICA is 1.00 which implies that the throughput achieved by e-TICA for the grid topology is equal to the maximum achievable throughput for that topology. As shown in Table 29 the fairness index of the Grid topology using e-TICA is 0.88 .

\subsubsection{Performance evaluation of e-TICA2}

As shown in Table 27 e-TICA2 also does not encounter any LICs for the Grid topology. As shown in Table 28 the throughput ratio of the Grid topology using e-TICA2 is 1 which implies that the throughput achieved by e-TICA2 for the grid topology is equal to the maximum achievable throughput for the topology. However, as shown in Table 29 the Jain's fairness index of the Grid topology using e-TICA2 is 0.58 which is lower than the Jain's fairness index of TICA and e-TICA. A lower fairness index for the grid topology using e-TICA2, implies that even though fairness in medium access has been achieved since there are no LICs present, per-flow fairness suffers since nine traffic 
sources share the link 15-9 and the other three links have one source each. This results in congestion on the link 15-9 hence reducing the per flow fairness.

Table 27: Number of LICs found in grid topology

\begin{tabular}{|c|r|r|r|}
\hline & TICA & e-TICA & e-TICA2 \\
\hline Grid Topology & 0 & 0 & 0 \\
\hline
\end{tabular}

Table 28: Throughput ratio for the grid topology

\begin{tabular}{|c|c|c|c|}
\hline & TICA & e-TICA & e-TICA2 \\
\hline Grid Topology & 1.00 & 1.00 & 1.00 \\
\hline
\end{tabular}

Table 29: Jain's Fairness Index for grid topology

\begin{tabular}{|c|c|c|c|}
\hline & TICA & e-TICA & e-TICA2 \\
\hline Grid Topology & 0.88 & 0.88 & 0.58 \\
\hline
\end{tabular}




\subsection{Consolidation of the results:}

Table 30, Table 31 and Table 32 consolidate the results for all three channel assignment algorithms.

Table 30: 95\% confidence intervals for Number of LICs

\begin{tabular}{|l|c|c|c|}
\hline CAA & Ropology & Controlled Random & Grid \\
\hline TICA & $1.79-2.69$ & $2.89-4.23$ & 0 \\
\hline e-TICA & $2.38-3.62$ & $3.40-4.92$ & 0 \\
\hline e-TICA2 & $1.36-2.08$ & $0.82-1.66$ & 0 \\
\hline
\end{tabular}

Table 31: 95\% confidence intervals for Throughput Ratio

\begin{tabular}{|l|c|c|c|}
\hline CAA & Ropology & Condrolled Random & Grid \\
\hline TICA & $0.77-0.91$ & $0.84-0.93$ & 1.00 \\
\hline e-TICA & $0.83-0.97$ & $0.90-0.97$ & 1.00 \\
\hline e-TICA2 & $0.89-0.98$ & $0.90-0.98$ & 1.00 \\
\hline
\end{tabular}

Table 32: 95\% confidence intervals for Fairness Ratio

\begin{tabular}{|l|c|c|c|}
\hline CAA & Ropology & Condom & Grid \\
\hline e-TICA over TICA & $1.02-1.14$ & $1.01-1.11$ & 1 \\
\hline e-TICA2 over e-TICA & $0.91-1.06$ & $0.84-1.01$ & 0.66 \\
\hline e-TICA2 over TICA & $0.97-1.13$ & $0.89-1.05$ & 0.66 \\
\hline
\end{tabular}




\section{Chapter - 6 : Conclusion and Future Work}

\subsection{Conclusions}

The enhancements made to TICA are the following:

1. A More accurate Channel assignment algorithm: The key objective during the channel assignment phase in an MRMC WMN is to eliminate the presence of interfering channels within the interference range of nodes. However, due to the availability of a limited number of orthogonal channels, this is not always possible. Hence, a CAA which reduces interference among nodes and provides maximum spatial reuse is needed. In this thesis, we introduced e-TICA which uses 2-way interference range edge coloring model. e-TICA provides a more accurate channel assignment strategy.

2. ReduCing InTERfERENCE: We also introduced e-TICA2 to reduce co-channel interference problems caused by long links in a random topology. e-TICA2 utilizes an MST rooted at the GW. The shorter links resulting from MST lead to a small interference range. Replacing SPT with MST in e-TICA2 leads to a reduction in the occurrence of LICs, which reduces interference and improves network throughput without compromising the network fairness.

3. Reducing CONGESTION: e-TICA2 helps in reducing traffic congestion at the gateway by utilizing the maximum possible radios of the GW. This helps in distributing the traffic load among the four links of the GW.

\subsection{Discussion}

- e-TICA2 gives a better performance among all three CAAs in terms of throughput ratio and fairness ratio for random topology.

- e-TICA and TICA give better results among all three CAAs in terms of both throughput ratio and fairness ratio for the grid topology. 
- The performance of e-TICA2 and e-TICA is comparable for controlled random topologies. This is due to low randomness in controlled random topologies; there is less difference between the shortest path tree and minimum spanning tree. Thus, the link lengths are almost the same.

\subsection{Trade-offs}

- Since the minimum spanning tree approach replaces the long links of shortest path tree with shorter ones, it means that the number of hops from each source to the GW increases. This implies a greater delay.

\subsection{Future Work}

- Since the maximum achievable throughput of a topology is limited by the number of traffic sources and the number of links emanating from the gateway, the use of multiple gateways for even distribution of network traffic should reduce traffic congestion and is expected to enhance the network performance.

- Position of the gateway plays a vital role in the performance of the algorithm. If the gateway is centrally placed between the nodes, the distribution of traffic will be even which should eventually lead to better network performance.

- The free space propagation model and the two-ray propagation models have been used in this thesis. As future work, a more realistic shadowing model could be used to verify the effectiveness of the algorithms.

- The formula used for calculating the interference level of a channel could be modified to reflect the number of times a channel has been used as an LIC. This would help in mitigating flow starvation in a case where the same link has to contend with two other links. Also, channels used for links emanating from the gateway and having many traffic sources on them, should be avoided as LICs since this causes all the sources on that particular link to suffer. 


\section{References}

[1] E. Hossain and K. Leung, Wireless Mesh Networks: Architectures and Protocols. US: Springer, 2007.

[2] I.F. Akyildiz, X. Wang, and W. Wang, "Wireless mesh networks: a survey," Computer Networks Journal, vol. 47 no. 4, pp. 445-487, 2005.

[3] "IEEE Standard for Information Technology-Telecommunications and Information Exchange Between Systems-Local and Metropolitan Area Networks-Specific Requirements - Part 11: Wireless LAN MAC and PHY Specifications," IEEE Std 802.11-2007 (Revision of IEEE Std 802.11-1999), Jun. 2007.

[4] S. S. Ghosh, S. K. Das, L. Lenzini, and M. Conti, "Channel assignment strategies for multiradio wireless mesh networks: issues and solutions," IEEE Communications Magazine, vol. 45 no. 11, pp. 86-9, 2007.

[5] A. U. Chaudhry, R. H. M. Hafez, O. Aboul-Magd, and S. A. Mahmoud, "Throughput Improvement in Multi-Radio Multi-Channel 802.11a-based Wireless Mesh Networks," in Proceedings of IEEE Globecom 2010, Dec. 2010.

[6] M. Mahdian, "On the Computational Complexity of Strong Edge Coloring," Discrete Applied Mathematics, vol. 118, pp. 239-248, 2002.

[7] J. LaRocca and R. LaRocca, 802.11 Demystified:Wi-Fi Made Easy. US: Tata McGraw Hill Professional, 2002.

[8] N. Nandiraju, D. Nandiraju, L. Santhanam, B. He, J. Wang, and D.P. Agrawal, "Wireless Mesh Networks: Current Challenges and Future Directions of Web-in-theSky," IEEE Wireless Communications Magazine, vol. 14 no.4, pp. 79-89, Aug. 2007.

[9] H. Wu and Y. Pan, Medium Access Control in Wireless Networks, New York, US: Nova Science Publishers, 2008.

[10] A. K Talukder and R. Yavagal, Mobile Computing, New Delhi, India: Tata McGraw-Hill, 2005.

[11] R. Koshy and R. Lu, "A Joint Radio and Channel Assignment (JRCA) Scheme for 802.11-Based Wireless Mesh Networks," in Proceedings of IEEE GLOBECOM Workshops, 2009.

[12] H. M. Ali, A. Busso, and V. Veque, "Channel assignment algorithms: a comparison of graph based heuristics," in Proceedings of ACM PM2HW2N 2009, pp. 120-127, Oct. 2009.

[13] S. Avallone and I.F. Akyildiz. "A channel assignment algorithm for multi-radio wireless mesh networks". Computer Communications, vol. 31 no.7, pp. 1343-1353, May 2008. 
[14] A. P. Subramanian, H. Gupta, S. R. Das, and C. Jing , "Minimum Interference Channel Assignment in Multiradio Wireless Mesh Networks," IEEE Transactions on Mobile Computing, vol.7, no.12, pp.1459-1473, Dec. 2008.

[15] K. N. Ramachandran, E. M. Belding, K. C. Almeroth, and M. M. Buddhikot, "Interference-Aware Channel Assignment in Multi-Radio Wireless Mesh Networks," in Proceedings of IEEE INFOCOM 2006, April 2006.

[16] H. Huang, X. Cao, and X. Wang, "Topology simplification and channel assignment in multi-radio Wireless Mesh Networks," IEEE International Conference on Systems, Man and Cybernetics, 2008. SMC 2008.

[17] M.K. Marina and S.R. DAS, "A Topology Control Approach for Utilizing Multiple Channels in Multi-radio wireless Mesh Networks," The 2nd International Conference on Broadband Networks, 2005

[18] K. Athota, A. Negi, and C. R. Rao, "Interference-traffic aware Channel Assignment for MRMC WMNs," IEEE 2nd International Advance Computing Conference (IACC), 2010 , pp.273-278, 19-20 Feb. 2010

[19] N. Li, J. Hou, and L. Sha, "Design and analysis of an MST-based topology control algorithm," in Proceedings of INFOCOM, IEEE International Conference, 2003.

[20] X. Wang and K. Kar, "Throughput modeling and fairness issues in CSMA/CA based ad-hoc networks," in Proceedings of 24th Annual Joint Conference of the IEEE Computer and Communications Societies (INFOCOM 2000), vol.1, pp. 23-34, 13-17 March 2005

[21] L. B. Jiang and S. C. Liew, "Improving Throughput and Fairness by Reducing Exposed and Hidden Nodes in 802.11 Networks," IEEE Transactions on Mobile Computing, vol.7, no.1, pp.34-49, Jan. 2008

[22] D.D. Vergados, D. J. Vergados, A. Sgora, D. Vouyioukas, and I. Anagnostopoulos, "Enhancing fairness in wireless multi-hop networks," in Proceedings of the 3rd international conference on Mobile multimedia communications (MobiMedia '07), Article 37.

[23] A. Mishra, V. Shrivastava, D. Agrawal, S. Banerjee, and S. Ganguly, 2006. "Distributed channel management in uncoordinated wireless environments," in Proceedings of the 12th annual international conference on Mobile computing and networking (MobiCom '06). ACM, New York, NY, USA, 170-181.

[24] M. Durvy, O. Dousse, and P. Thiran, "Self-Organization Properties of CSMA/CA Systems and Their Consequences on Fairness," IEEE Transactions on Information Theory, vol.55, no.3, pp.931-943, March 2009

[25] C. Huang, C. Lea, and A. K.-S. Wong, "On Fairness Enhancement for CSMA/CA Wireless Networks," IEEE Systems Journal, vol.4, no.4, pp.51 1-523, Dec. 2010

[26] R. Jain, The Art of Computer Systems Performance Analysis: Techniques for Experimental Design, Measurement, Simulation, and Modeling. New York: WileyInterscience, 1991. 


\section{Appendix-A: SPT for Random Topologies}
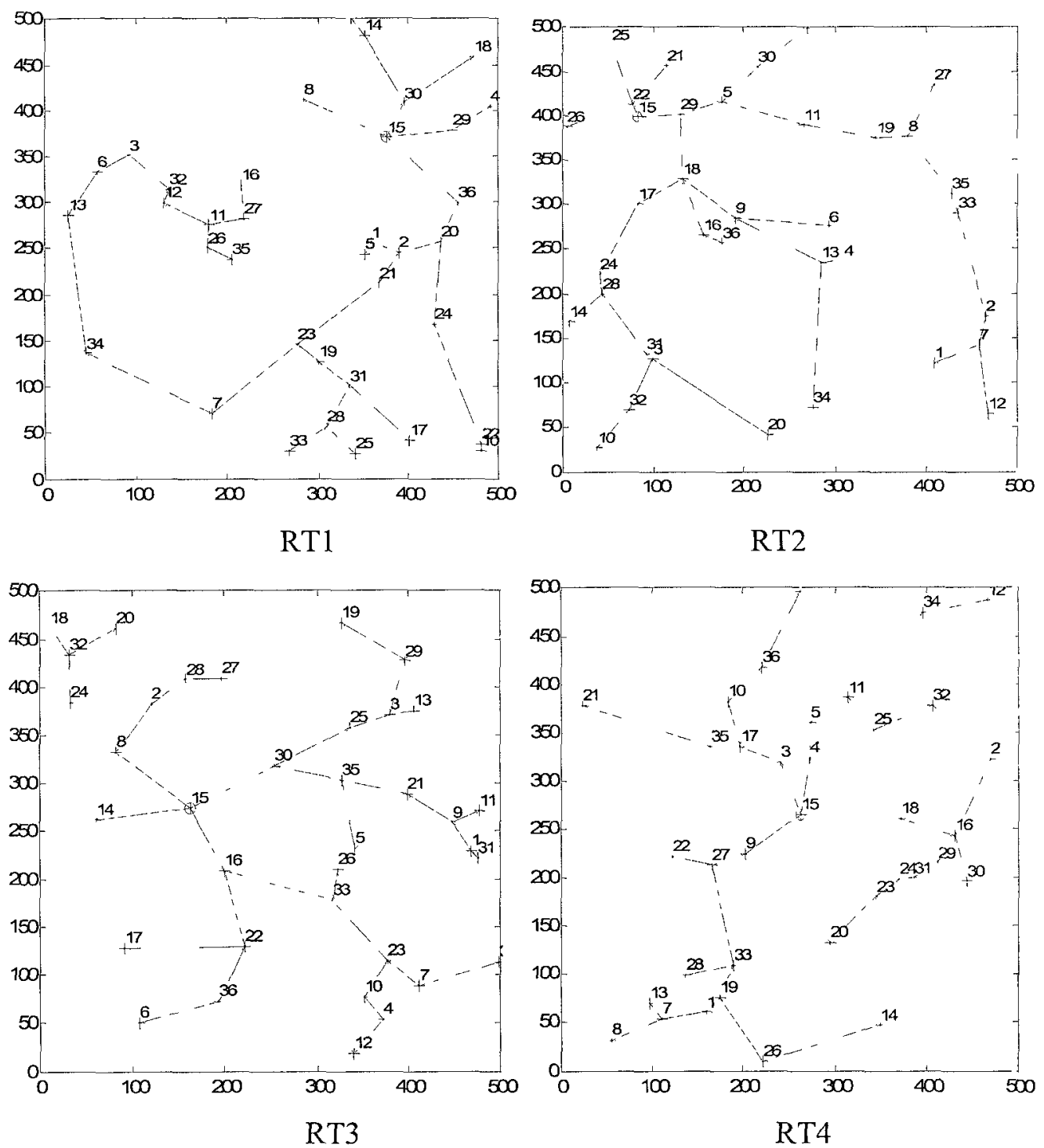


\section{Appendix-B: SPT for Controlled Random Topologies}
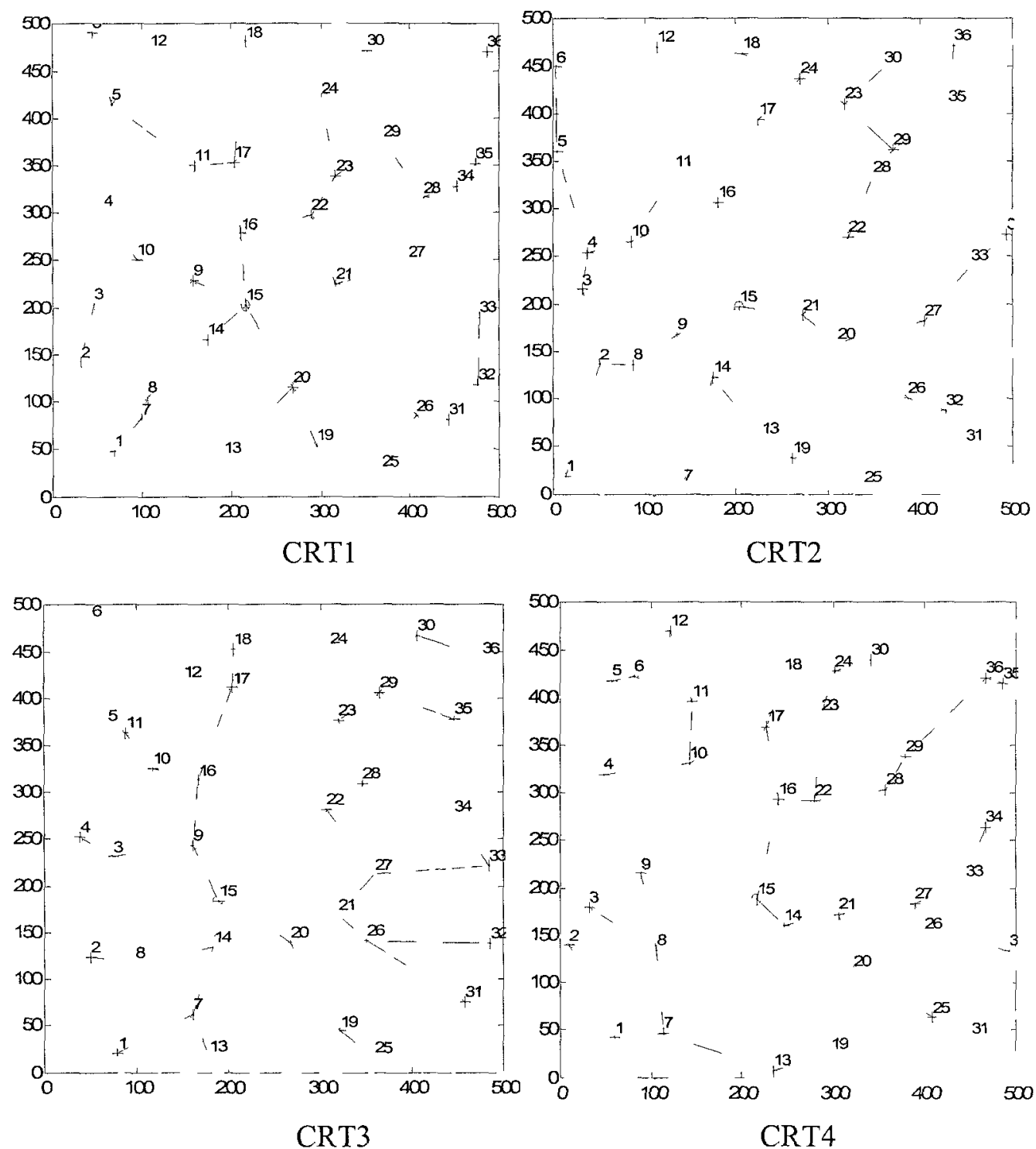


\section{Appendix-C: MST for Random Topologies}
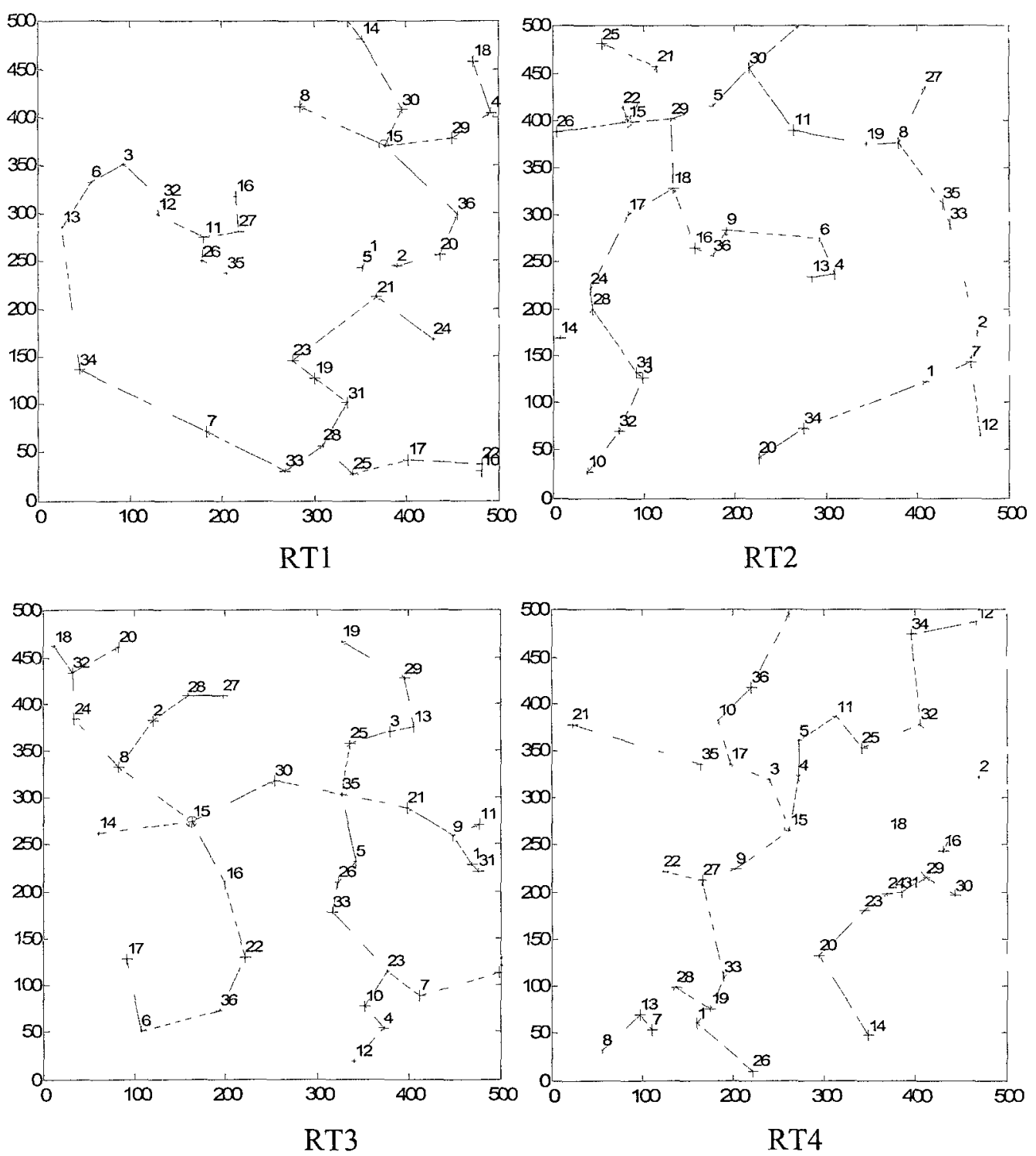


\section{Appendix-D: MST for Controlled Random Topologies}
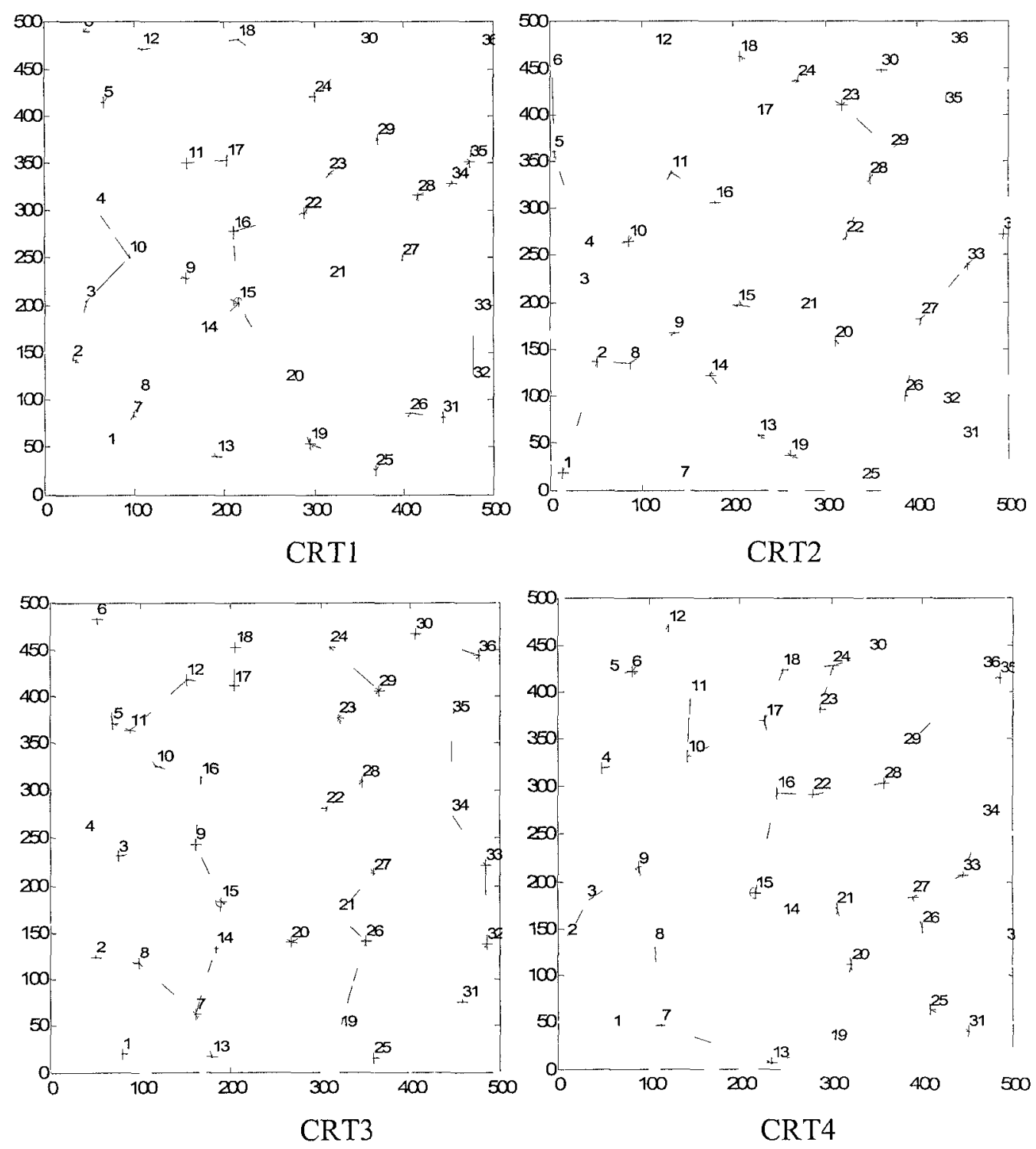


\section{Appendix-E: SPT and MST for Grid Topology}
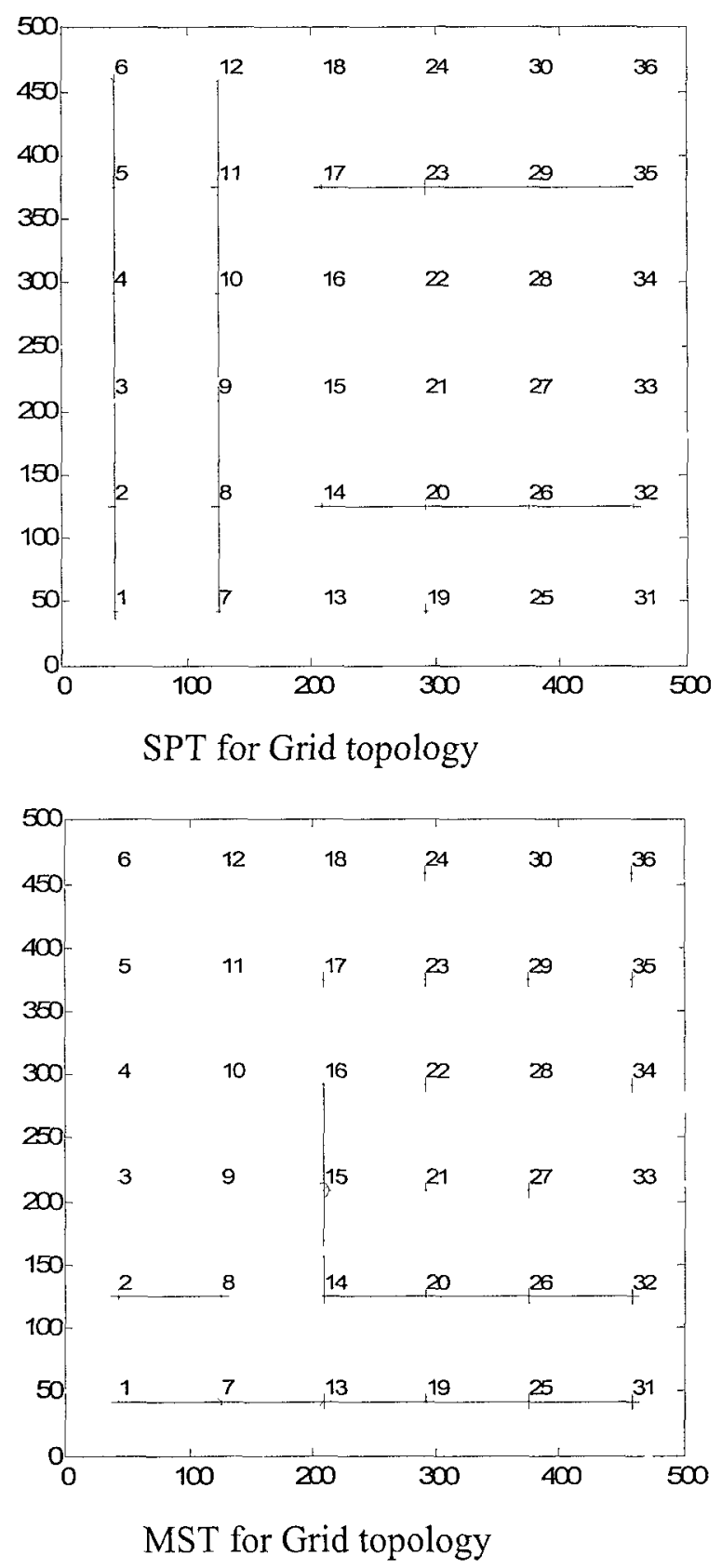